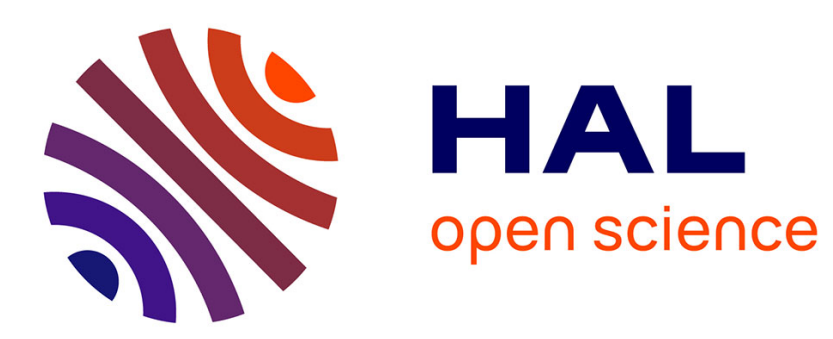

\title{
Resolving power of MUSIC-like algorithms for circular or rectilinear correlated sources in CES data models
}

Habti Abeida, Jean-Pierre Delmas

\section{To cite this version:}

Habti Abeida, Jean-Pierre Delmas. Resolving power of MUSIC-like algorithms for circular or rectilinear correlated sources in CES data models. Signal Processing, 2021, 188, pp.108234:1-108234:14. 10.1016/j.sigpro.2021.108234 . hal-03366804

\section{HAL Id: hal-03366804 https://hal.science/hal-03366804}

Submitted on 5 Oct 2021

HAL is a multi-disciplinary open access archive for the deposit and dissemination of scientific research documents, whether they are published or not. The documents may come from teaching and research institutions in France or abroad, or from public or private research centers.
L'archive ouverte pluridisciplinaire HAL, est destinée au dépôt et à la diffusion de documents scientifiques de niveau recherche, publiés ou non, émanant des établissements d'enseignement et de recherche français ou étrangers, des laboratoires publics ou privés. 


\title{
Resolving power of MUSIC-like algorithms for circular or rectilinear correlated sources in CES data models
}

\author{
Habti Abeida and Jean-Pierre Delmas
}

\begin{abstract}
The concept of threshold array signal-to-noise ratio (ASNR) which is defined as the minimal SNR at which specific high-resolution algorithms are able to resolve two closely spaced far-field sources, allows to quantify and to compare sensors array performance in localizing remote targets. This paper generalizes and extends the expressions of the threshold ASNR given in the literature for the conventional and non-circular (NC) MUSIC direction-of-arrival (DOA) estimation algorithms in the context of uncorrelated stochastic circular or rectilinear Gaussian sources and circular complex Gaussian (C-CG) noise, in a more general stochastic framework. We assume that the sources are correlated with an arbitrary distribution, which is inherent in a context of multipath or smart jammers, and that the noise is circular complex elliptically symmetric (C-CES) distributed, which can model impulsive noise with heavy-tailed distributions. The C-CES and NC-CES distributed observations are also considered to quantify the gain in resolution provided when the sample covariance matrix (SCM) of the observations is replaced by $M$-estimates of this matrix. Asymptotic approaches and perturbation analysis have been performed to derive closed-form expressions of the mean null spectra of the two considered MUSIC algorithms for both observation models, which allow us to derive, for the first time, general unified explicit analytical expressions of the threshold ASNR along the Cox and the Sharman and Durrani criteria. These expressions allow us to quantify the impact of the non-Gaussianity of noise and observations, as well as of the phase and magnitude of the correlation on the resolution threshold, and to quantify the benefit provided when the SCM is replaced by $M$-estimates of this covariance matrix for CES observations. Finally, numerical illustrations are included to support our theoretical analysis.
\end{abstract}

\section{Index Terms}

Habti Abeida is with Dept. of Electrical Engineering, University of Taif, Al-Haweiah, 21974, Saudi Arabia, email: h.abeida@tu.edu.sa, phone: +(33).1.60.76.46.32. Jean-Pierre Delmas is with Samovar laboratory, Telecom SudParis, Institut Polytechnique de Paris, 91011 Evry Cedex, France, e-mail: jean-pierre.delmas@it-sudparis.eu, phone: +(33).1.60.76.46.32, fax: +(33).1.60.76.44.33. 
Direction of arrival (DOA), circular/noncircular sources, rectilinear, strictly second-order noncircular, circular/noncircular MUSIC algorithms, circular/noncircular CES distributions, threshold ASNR, resolution limit.

Paper accepted to Signal Processing

\section{INTRODUCTION}

The ability to resolve two closely-spaced narrowband far-field sources is an important performance measure of sensor arrays in localizing remote targets with wide-ranging application in, among others, astronomy, radar and wireless communications [1]. This very old problem has been extensively studied in the literature and is still the object of active research in many applications (see e.g., [2], [3]). There are usually four different approaches in the literature to determine the resolution limit of two closely spaced signals. The first one rests on the analysis of the mean null spectrum concerning specific high-resolution algorithms. More precisely, two main criteria based on the mean null spectrum have been introduced by Cox [4] and Sharman and Durrani [5]. The first criterion states that two sources are resolved if the midpoint mean null spectrum is greater than the mean null spectrum in the two true source DOAs. While for the second criterion, two sources are resolved if the second derivative of the mean spectrum at the midpoint is negative. The two criteria were applied to derive the threshold ASNR for specific high-resolution algorithms, including conventional MUSIC and Min-Norm (the first one in [6]-[10] and the second one in [11], [12]). Then, they have been used to compare the threshold ASNR for the conventional MUSIC and NC MUSIC algorithms [13] in the context of uncorrelated circular and rectilinear sources, respectively. The second approach is based on a hypothesis test using the generalized likelihood ratio test [14], [15] or the Bayesian approach [16]. The third approach relies on the estimation accuracy, capitalizing on the Cramér-Rao bound (CRB). It compares the DOA separation of two sources to the square-root of the CRB of the DOA [17] or to the CRB of the difference between the two DOA's introduced in [18] and then used in numerous papers (see e.g., [19]-[22]). The last approach is based on the information theory and more specifically on the Stein's lemma which links the false alarm probability resulted from the Neyman-Pearson decision criterion to the relative entropy between two hypothesis [23], or on the mutual information between DOA, scattering properties and the received signal [2]. In all these works, however, the sources embedded in a spatially uncorrelated C-CG distributed noise are considered either deterministic or uncorrelated stochastic Gaussian distributed, except in [9] which presents some simulation results using the null spectrum with correlated and coherent C-CG distributed sources, and in [22] which uses the Smith's criterion [18] in the MIMO radar context under $K$-distributed clutter.

This paper is dedicated to the threshold ASNRs derivation based on the first approach and its aim is twofold. First, it gives theoretical approximate interpretable closed-form expressions of the threshold ASNR for which 
MUSIC-like algorithms are able to resolve two closely spaced sources in a much more general statistical framework than in the previous works. More precisely, we assume here that the directional sources are circular or rectilinear correlated with arbitrary distributions, which is inherent in a context of multipath or smart jammers. We also consider that the noise is spatially uncorrelated and C-CES distributed, which can model impulsive noise with heavy-tailed distributions. This kind of noise can be encountered in radar clutter [24] [25], mademan noise and interference in indoor and outdoor mobile communications channels [26] [27]. In these adverse conditions, this allows us, in particular, to quantify the increase of threshold ASNR required to resolve two closely-spaced equipowered sources with the conventional MUSIC and NC MUSIC algorithms based on the SCM of the observations, with respect to the standard conditions of uncorrelated sources embedded in C-CG distributed noise. Second, it aims is to quantify the gain in resolution brought when this SCM is replaced by an $M$-estimate of the covariance matrix of the observations. For this purpose, interpretable closed-form expressions of the threshold ASNR are also derived for C-CES and NC-CES distributed observations.

The paper is organized as follows. Section II gives a brief reminder on CES distribution and specifies the data models and the involved MUSIC-based DOA estimation algorithms. It first describes a standard array data model with two equal-power arbitrary distributed correlated sources, which are either circular or rectilinear, and a spatially white C-CES distributed noise. Then, a robust distribution model, where the observations are either C-CES or NC-CES distributed, is introduced. This section ends with a brief review of the conventional and NC MUSIC algorithms and the statistical distributions of the SCM and $M$-estimators of covariance matrices. Section III presents the resolving power of MUSIC-like algorithms. After a brief review of the Cox, and of Sharman and Durrani criteria on which resolving power is based, two preliminary lemmas based on perturbations of noise projectors are given. They allow us to give closed-form expressions of the mean-null spectrum associated with the conventional and NC MUSIC algorithms for the two models introduced in Section II. Two general closed-form expressions of the threshold ASNR applicable to the two models and criteria for each conventional MUSIC and NC MUSIC algorithms are deduced. Then, comments to explain how the non-Gaussianity of the noise and observations, and the phase and magnitude of the correlation of the sources impact the threshold ASNR are discussed. Numerical illustrations of the threshold ASNR are given in Section IV, with particular attention paid to the phase and magnitude of the correlation of the sources and to the robustness of $M$-estimates of covariance matrices of the observations. Finally, the paper is concluded in Section V.

The notations used throughout this paper are the following. The abbreviation [resp. ] stands for respectively. Vectors and matrices are denoted by bold-faced lowercase and uppercase letters, respectively. ${ }^{*},{ }^{T}$, and ${ }^{H}$ respectively represent the conjugate, the transpose and the conjugate transpose operators. $\mathrm{E}(),. \operatorname{Re}(),.|$.$| and$ \# are the expectation, real part operator, determinant and Moore-Penrose inverse, respectively. $={ }_{d}$ stands for 
"shares the same distribution as". $\operatorname{vec}(\cdot)$ is the vectorization operator that turns a matrix into a vector by stacking the columns of the matrix one below another which is used in conjunction with the Kronecker product $\mathbf{A} \otimes \mathbf{B}$ as the block matrix whose $(i, j)$ block element is $a_{i, j} \mathbf{B}$ and with the vec-permutation matrix $\mathbf{K}$ which transforms $\operatorname{vec}(\mathbf{C})$ to $\operatorname{vec}\left(\mathbf{C}^{T}\right)$ for any matrix $\mathbf{C}$. The matrix $\mathbf{J}$ is the exchange matrix $\left(\begin{array}{ll}\mathbf{0} & \mathbf{I} \\ \mathbf{I} & \mathbf{0}\end{array}\right)$ and $\mathbf{e}_{k}$ denotes the $k$-unit vector.

\section{DATA MODEL AND MUSIC-LIKE ALGORITHMS}

In this section, we specify two data array models with two equal-power arbitrary distributed correlated sources impinging on an arbitrary array of $N$ sensors with spatially white noise. In the first model, the sources are either arbitrary or rectilinear with a C-CES distributed noise and in the second one, the observation is either C-CES or NC-CES distributed. For the ease of the readers, we begin by giving a brief reminder on C-CES and NC-CES distributions.

\section{A. Brief review of $C$-CES and NC-CES distributions}

In this paper, we use $N$-dimensional zero-mean C-CES [resp., NC-CES] distributed random variables (r.v.) $\mathbf{y}_{t}$ possessing probability density functions (p.d.f.) given by

$$
\left.p\left(\mathbf{y}_{t}\right)=\left|\mathbf{R}_{y}\right|^{-1} g\left(\mathbf{y}_{t}^{H} \mathbf{R}_{y}^{-1} \mathbf{y}_{t}\right), \quad \text { [resp., } \quad\left|\mathbf{R}_{\tilde{y}}\right|^{-1 / 2} g\left(\frac{1}{2} \tilde{\mathbf{y}}_{t}^{H} \mathbf{R}_{\tilde{y}}^{-1} \tilde{\mathbf{y}}_{t}\right)\right],
$$

where $\tilde{\mathbf{y}}_{t} \stackrel{\text { def }}{=}\left[\mathbf{y}_{t}^{T}, \mathbf{y}_{t}^{H}\right]^{T}$ and $\mathbf{R}_{\tilde{y}} \stackrel{\text { def }}{=}\left(\begin{array}{cc}\mathbf{R}_{y} & \mathbf{C}_{y} \\ \mathbf{C}_{y}^{*} & \mathbf{R}_{y}^{*}\end{array}\right)$ with $\mathbf{R}_{y}$ and $\mathbf{C}_{y}$ are $N \times N$ Hermitian positive definite and complex symmetric matrices, respectively called scatter and pseudo-scatter matrices. The function ${ }^{1} g($.$) :$ $\mathbb{R}^{+} \mapsto \mathbb{R}^{+}$satisfies $\delta_{N, g} \stackrel{\text { def }}{=} \int_{0}^{\infty} t^{N-1} g(t) d t<\infty$. The r.v. $\mathbf{y}_{t}$ admit the following stochastic representation: $\mathbf{y}_{t}={ }_{d} \sqrt{\mathcal{Q}_{t}} \mathbf{R}_{y}^{1 / 2} \mathbf{u}_{t}$ [28] [resp., $\tilde{\mathbf{y}}_{t}={ }_{d} \sqrt{\mathcal{Q}_{t}} \mathbf{R}_{\tilde{y}}^{1 / 2} \tilde{\mathbf{u}}_{t}$ [29] with $\tilde{\mathbf{u}}_{t} \stackrel{\text { def }}{=}\left[\mathbf{u}_{t}^{T}, \mathbf{u}_{t}^{H}\right]^{T}$ ], where the non-negative real r.v. $\mathcal{Q}_{t}$ and the complex r.v. $\mathbf{u}_{t}$ are independent and $\mathbf{u}_{t}$ is uniformly distributed on the unit complex $N$-sphere.

\section{B. Standard data model}

Consider two equal-power narrowband uncorrelated or correlated (but non-coherent) signals impinging on an arbitrary array of $N$ sensors. The signal received in baseband at the time instant $t$ is modeled as

$$
\mathbf{y}_{t}=\mathbf{A} \mathbf{x}_{t}+\mathbf{n}_{t}, \quad t=1, \ldots, T
$$

\footnotetext{
${ }^{1}$ To remove the so-called scale ambiguity, the density generator $g$ is here constrained such that $\delta_{N+1, g} / \delta_{N, g}=N$, [28] to ensure that the scatter $\mathbf{R}_{y}$ and extended scatter $\mathbf{R}_{\tilde{y}}$ matrices are equal to the covariance $\mathrm{E}\left(\mathbf{y}_{t} \mathbf{y}_{t}^{H}\right)$ and extended covariance $\mathrm{E}\left(\tilde{\mathbf{y}}_{t} \tilde{\mathbf{y}}_{t}^{H}\right)$, respectively. The expressions of these p.d.f. are consistent with the ones given in [28] and [30], respectively, because the normalizing constant is here included in the function $g$.
} 
where $\left\{\mathbf{y}_{1}, \ldots, \mathbf{y}_{t}, \ldots, \mathbf{y}_{T}\right\}$ are independent and identically distributed. $\mathbf{A}=\left[\mathbf{a}_{1}, \mathbf{a}_{2}\right]$ is the steering matrix where each vector $\mathbf{a}_{k}=\mathbf{a}\left(\theta_{k}\right)$ is parameterized by the real scalar parameter $\theta_{k}$ according to the parametrization introduced in [10] where $\left\|\mathbf{a}_{k}\right\|^{2}=N . \mathbf{x}_{t}=\left[x_{t, 1}, x_{t, 2}\right]^{T}$ and $\mathbf{n}_{t}$ model signals transmitted by sources and additive measurement noise, respectively. $\mathbf{x}_{t}$ and $\mathbf{n}_{t}$ are zero-mean with finite fourth-order moments and not correlated with each other. $\mathbf{n}_{t}$ is assumed to be C-CES distributed and spatially uncorrelated with $\mathrm{E}\left(\mathbf{n}_{t} \mathbf{n}_{t}^{H}\right)=\sigma_{n}^{2} \mathbf{I}$. The fourth-order moments of $\mathbf{n}_{t}$ are proved to be characterized by a single parameter $\eta$. This parameter is defined by $\eta \stackrel{\text { def }}{=} \frac{\mathrm{E}\left(\mathcal{Q}_{t}^{2}\right)}{N(N+1)}$ from the stochastic representation $\mathbf{n}_{t}={ }_{d} \sigma_{n} \sqrt{\mathcal{Q}_{t}} \mathbf{u}_{t}$. This parameter satisfies the conditions $\eta \geq N /(N+1)$ [31], $\eta=1$ for C-CG distributions and can take very large values for very impulsive noise, such that for the circular complex Student $t$-distributions with heavier tails than the C-CG distribution as shown in Section IV-A. The signal sources $\mathbf{x}_{t}$ are arbitrary distributed (circular or non-circular to the second-order), with covariance $\mathbf{R}_{x}=\mathrm{E}\left(\mathbf{x}_{t} \mathbf{x}_{t}^{H}\right)$ and complementary covariance $\mathbf{C}_{x}=\mathrm{E}\left(\mathbf{x}_{t} \mathbf{x}_{t}^{T}\right)$. Consequently, this leads to the following covariance and complementary covariance of $\mathbf{y}_{t}$

$$
\mathbf{R}_{y}=\mathbf{A} \mathbf{R}_{x} \mathbf{A}^{H}+\sigma_{n}^{2} \mathbf{I} \stackrel{\text { def }}{=} \mathbf{S}+\sigma_{n}^{2} \mathbf{I} \text { and } \mathbf{C}_{y}=\mathbf{A} \mathbf{C}_{x} \mathbf{A}^{T}
$$

where

$$
\mathbf{R}_{x}=\sigma_{x}^{2}\left(\begin{array}{cc}
1 & \rho \\
\rho^{*} & 1
\end{array}\right) \text { with } \rho \stackrel{\text { def }}{=} \mathrm{E}\left(x_{t, 1} x_{t, 2}^{*}\right) \in \mathbb{C} \text { and }|\rho|<1 .
$$

We will also consider the specific case where $x_{t, 1}$ and $x_{t, 2}$ are rectilinear (also called strictly second-order non-circular), i.e., described by

$$
x_{t, k}=s_{t, k} e^{i \phi_{k}} \text { where } s_{t, k} \text { are real-valued with } \Delta \phi \stackrel{\text { def }}{=} \phi_{1}-\phi_{2} \in[0,+\pi] .
$$

The phases $\phi_{k}$ associated with different propagation delays are assumed fixed, but unknown during the array observation. To derive MUSIC-like algorithms exploiting the prior knowledge of rectilinear sources, the model (2) can be rewritten according to the following equivalent extended model:

$$
\widetilde{\mathbf{y}}_{t} \stackrel{\text { def }}{=}\left[\begin{array}{c}
\mathbf{y}_{t} \\
\mathbf{y}_{t}^{*}
\end{array}\right]=\widetilde{\mathbf{A}} \mathbf{s}_{t}+\widetilde{\mathbf{n}}_{t}, \quad t=1, \ldots, T,
$$

where $\widetilde{\mathbf{A}} \stackrel{\text { def }}{=}\left[\widetilde{\mathbf{a}}_{1}, \widetilde{\mathbf{a}}_{2}\right]$ with $\widetilde{\mathbf{a}}_{k} \stackrel{\text { def }}{=}\left[\mathbf{a}_{k}^{T} e^{i \phi_{k}}, \mathbf{a}_{k}^{H} e^{-i \phi_{k}}\right]^{T}, \mathbf{s}_{t} \stackrel{\text { def }}{=}\left[s_{t, 1}, s_{t, 2}\right]^{T}$ and $\widetilde{\mathbf{n}}_{t} \stackrel{\text { def }}{=}\left(\mathbf{n}_{t}^{T}, \mathbf{n}_{t}^{H}\right)^{T}$. Consequently the covariance $\mathbf{R}_{\tilde{y}} \stackrel{\text { def }}{=} \mathrm{E}\left(\widetilde{\mathbf{y}}_{t} \widetilde{\mathbf{y}}_{t}^{H}\right)$ and complementary covariance $\mathbf{C}_{\tilde{y}} \stackrel{\text { def }}{=} \mathrm{E}\left(\widetilde{\mathbf{y}}_{t} \widetilde{\mathbf{y}}_{t}^{T}\right)$ of the extended signal $\widetilde{\mathbf{y}}_{t}$ are given by

$$
\mathbf{R}_{\tilde{y}}=\widetilde{\mathbf{A}} \mathbf{R}_{s} \widetilde{\mathbf{A}}^{H}+\sigma_{n}^{2} \mathbf{I} \stackrel{\text { def }}{=} \widetilde{\mathbf{S}}+\sigma_{n}^{2} \mathbf{I} \text { and } \mathbf{C}_{\tilde{y}}=\mathbf{R}_{\tilde{y}} \mathbf{J}=\mathbf{J} \mathbf{R}_{\tilde{y}}^{*}
$$


where if

$$
\mathbf{R}_{s} \stackrel{\text { def }}{=} \mathrm{E}\left(\mathbf{s}_{t} \mathbf{s}_{t}^{T}\right)=\sigma_{x}^{2}\left(\begin{array}{cc}
1 & \rho^{\prime} \\
\rho^{\prime} & 1
\end{array}\right) \text { with } \rho^{\prime} \in(-1,+1),
$$

$\mathbf{R}_{x}$ in (3) is written in the following form:

$$
\mathbf{R}_{x}=\sigma_{x}^{2}\left(\begin{array}{cc}
1 & \rho^{\prime} e^{i \Delta \phi} \\
\rho^{\prime} e^{-i \Delta \phi} & 1
\end{array}\right)
$$

Consequently, in the specific case of rectilinear sources, the phase separation $\Delta \phi$ associated with the sign of $\rho^{\prime}$ corresponds to the phase $\angle \rho$ of the correlation of the sources.

Assuming that $\mathbf{A}$ and $\widetilde{\mathbf{A}}$ are full column rank, whose column spaces characterize the DOA $\left(\theta_{1}, \theta_{2}\right)$, the conventional and NC MUSIC algorithms are usually based on the SCM and extended SCM $\mathbf{R}_{y, T}=\frac{1}{T} \sum_{t=1}^{T} \mathbf{y}_{t} \mathbf{y}_{t}^{H}$ and $\mathbf{R}_{\tilde{y}, T}=\frac{1}{T} \sum_{t=1}^{T} \widetilde{\mathbf{y}}_{t} \widetilde{\mathbf{y}}_{t}^{H}$, respectively.

\section{Robust distribution model}

Many papers in the literature (see e.g., [28], [32]) have shown by numerical simulations that the MUSIC sample null spectrum presented a loss of resolution for heavy-tailed distributed noise. To mitigate this loss in resolution performance of MUSIC-like algorithms for heavy-tailed C-CES distributed noise, the SCM and extended SCM can be replaced by the ML estimate of $\mathbf{R}_{y}$ and $\mathbf{R}_{\tilde{y}}$, respectively. However, these ML estimates cannot be obtained for arbitrary distributed $\mathbf{x}_{t}$ and arbitrary C-CES distributed $\mathbf{n}_{t}$ in (2). To overcome this difficulty, we consider here an alternative model used in [31], [29] and [33], where the observations $\mathbf{y}_{t}$ in (2) are CES distributed. In this case the distributions of $\mathbf{x}_{t}$ and $\mathbf{n}_{t}$ are not specified, but only their second-order statistics are imposed. More specifically, in the cases where the signals $\mathbf{x}_{t}$ are circular to the second-order [resp., rectilinear], where $\left\{\mathbf{y}_{1}, \ldots, \mathbf{y}_{t}, \ldots, \mathbf{y}_{T}\right\}$ are assumed independent zero-mean C-CES [resp., NC-CES] identically distributed whose p.d.f. are given by (1).

The ML estimate of $\mathbf{R}_{y}$ [resp., $\mathbf{R}_{\tilde{y}}$ ] in this model is solution of the implicit equation:

$$
\boldsymbol{\Gamma}_{y, T}=\frac{1}{T} \sum_{t=1}^{T} \psi\left(\mathbf{y}_{t}^{H} \boldsymbol{\Gamma}_{y, T}^{-1} \tilde{\mathbf{y}}_{t}\right) \mathbf{y}_{t} \tilde{\mathbf{y}}_{t}^{H}, \quad\left[\operatorname{resp} ., \boldsymbol{\Gamma}_{\tilde{y}, T}=\frac{1}{T} \sum_{t=1}^{T} \psi\left(\frac{1}{2} \tilde{\mathbf{y}}_{t}^{H} \boldsymbol{\Gamma}_{\tilde{y}, T}^{-1} \tilde{\mathbf{y}}_{t}\right) \tilde{\mathbf{y}}_{t} \tilde{\mathbf{y}}_{t}^{H}\right],
$$

where $\psi(t) \stackrel{\text { def }}{=}-\frac{1}{g(t)} \frac{d g(t)}{d t}$. Existence, uniqueness of the solution of (10) and convergence in probability of the sequence $\boldsymbol{\Gamma}_{y, T}$ to $\mathbf{R}_{y}$ have been proved in [28]. These properties have been extended in [29] to the sequence $\boldsymbol{\Gamma}_{\tilde{y}, T}$ which converges in probability to $\mathbf{R}_{\tilde{y}}$.

These estimates belong to the class of $M$-estimators of scatter matrices introduced by Maronna [34], where $\psi($.$) does not need to be related to the density generator of any particular C-CES/NC-CES distribution. Existence$ and uniqueness of the solution of (10) where $\psi($.$) is replaced by a function u($.$) have been proved in the real$ 
case, provided $u($.$) satisfies a set of general conditions (called Maronna conditions) stated in [34].$

$$
\boldsymbol{\Gamma}_{y, T}=\frac{1}{T} \sum_{t=1}^{T} u\left(\mathbf{y}_{t}^{H} \boldsymbol{\Gamma}_{y, T}^{-1} \tilde{\mathbf{y}}_{t}\right) \mathbf{y}_{t} \tilde{\mathbf{y}}_{t}^{H}, \quad\left[\operatorname{resp} ., \boldsymbol{\Gamma}_{\tilde{y}, T}=\frac{1}{T} \sum_{t=1}^{T} u\left(\frac{1}{2} \tilde{\mathbf{y}}_{t}^{H} \boldsymbol{\Gamma}_{\tilde{y}, T}^{-1} \tilde{\mathbf{y}}_{t}\right) \tilde{\mathbf{y}}_{t} \tilde{\mathbf{y}}_{t}^{H}\right],
$$

These conditions have been extended to the complex case in [35] and [28]. These sequences denoted by $\boldsymbol{\Gamma}_{y, T}^{u}$ and $\Gamma_{\tilde{y}, T}^{u}$ to specify their dependence in $u($.$) converge in probability to \sigma_{u} \mathbf{R}_{y}$ and $\sigma_{u} \mathbf{R}_{\tilde{y}}$, respectively where $\sigma_{u}$ given by [28, (46)] depends on $u($.$) and the C-CES [resp., NC-CES] distribution of \mathbf{y}_{t}$ [resp., $\tilde{\mathbf{y}}_{t}$ ]. Note that Tyler's $M$-estimator [36] is also solution of (11) with the specific weight $u(t)=\frac{N}{t}$, does not satisfy Maronna conditions. It is a distribution-free estimator within the family of C-CES/NC-CES distributions. However, it has been proved for real elliptically symmetric (RES) distributions in [36] and for C-CES distributions in [37], then extended to NC-CES distributions in [29], that after normalizing, the solutions $\Gamma_{y, T}^{u}$ and $\Gamma_{\tilde{y}, T}^{u}$ of (10) also converges in probability to $\sigma_{u} \mathbf{R}_{y}$ and $\sigma_{u} \mathbf{R}_{\tilde{y}}$, respectively with $\sigma_{u}=1$.

\section{MUSIC-like algorithms}

For the standard and robust distribution models, we consider the conventional MUSIC algorithm associated with $\mathbf{R}_{y, T}$ and $\boldsymbol{\Gamma}_{y, T}^{u}$, respectively. The DOAs estimated by this algorithm are given by the two smallest minima of the following so-called sample null spectra $g_{T}^{\operatorname{Alg}_{\mathrm{C}}}(\theta)$ and $g_{T}^{\operatorname{Alg}_{\mathrm{C}}^{\mathrm{u}}}(\theta)$ [38]:

$$
\widehat{\theta}_{k, T}^{\mathrm{Alg}}=\arg \min _{\theta} g_{T}^{\mathrm{Alg} g_{\mathrm{C}}}(\theta) \quad \text { and } \quad \widehat{\theta}_{k, T}^{\mathrm{Alg} \mathrm{u}}=\arg \min _{\theta} g_{T}^{\mathrm{Alg} \mathrm{u}}(\theta), \quad k=1,2
$$

with

$$
g_{T}^{\operatorname{Alg}_{\mathrm{C}}}(\theta) \stackrel{\text { def }}{=} \mathbf{a}^{H}(\theta) \boldsymbol{\Pi}_{T} \mathbf{a}(\theta) \text { and } g_{T}^{\operatorname{Alg}_{\mathrm{C}}^{\mathrm{u}}}(\theta) \stackrel{\text { def }}{=} \mathbf{a}^{H}(\theta) \boldsymbol{\Pi}_{T}^{u} \mathbf{a}(\theta)
$$

where $\boldsymbol{\Pi}_{T}$ and $\boldsymbol{\Pi}_{T}^{u}$ denote the projector matrix associated with the noise subspace of $\mathbf{R}_{y, T}$ and $\boldsymbol{\Gamma}_{y, T}^{u}$, respectively.

In the specific case where $\mathbf{x}_{t}$ is rectilinear, we consider for both models, the NC MUSIC algorithm devised in [39] associated with $\mathbf{R}_{\tilde{y}, T}$ and $\Gamma_{\tilde{y}, T}^{u}$, respectively. The DOAs are also estimated by the two smallest minima of $g_{T}^{\mathrm{Alg}_{\mathrm{NC}}}(\theta)$ and $g_{T}^{\mathrm{Alg}_{\mathrm{NC}}^{\mathrm{u}}}(\theta)$, respectively

$$
\widehat{\theta}_{k, T}^{\mathrm{Alg} g_{N C}}=\arg \min _{\theta} g_{T}^{\operatorname{Alg}_{\mathrm{NC}}}(\theta) \quad \text { and } \quad \widehat{\theta}_{k, T}^{\mathrm{Alg} g_{N C}^{\mathrm{u}}}=\arg \min _{\theta} g_{T}^{\mathrm{Alg}_{\mathrm{NC}}^{\mathrm{u}}}(\theta), \quad k=1,2
$$

with

$g_{T}^{\mathrm{Alg}_{\mathrm{NC}}}(\theta) \stackrel{\text { def }}{=}\left(\mathbf{a}^{H}(\theta) \boldsymbol{\Pi}_{1, T} \mathbf{a}(\theta)\right)^{2}-\left|\mathbf{a}^{T}(\theta) \boldsymbol{\Pi}_{2, T}^{*} \mathbf{a}(\theta)\right|^{2} \quad$ and $\quad g_{T}^{\operatorname{Alg}_{\mathrm{NC}}^{\mathrm{u}}}(\theta) \stackrel{\text { def }}{=}\left(\mathbf{a}^{H}(\theta) \boldsymbol{\Pi}_{1, T}^{u} \mathbf{a}(\theta)\right)^{2}-\left|\mathbf{a}^{T}(\theta) \boldsymbol{\Pi}_{2, T}^{u *} \mathbf{a}(\theta)\right|^{2}$,

where $\boldsymbol{\Pi}_{1, T}$ and $\boldsymbol{\Pi}_{2, T}$ [resp., $\boldsymbol{\Pi}_{1, T}^{u}$ and $\left.\boldsymbol{\Pi}_{2, T}^{u}\right]$ are Hermitian and complex symmetric respectively, given by the 
projector matrices $\widetilde{\boldsymbol{\Pi}}_{T}$ and $\widetilde{\boldsymbol{\Pi}}_{T}^{u}$ structured as

$$
\left(\begin{array}{ll}
\boldsymbol{\Pi}_{1, T} & \boldsymbol{\Pi}_{2, T} \\
\boldsymbol{\Pi}_{2, T}^{*} & \boldsymbol{\Pi}_{1, T}^{*}
\end{array}\right) \text { and }\left(\begin{array}{cc}
\boldsymbol{\Pi}_{1, T}^{u} & \boldsymbol{\Pi}_{2, T}^{u} \\
\boldsymbol{\Pi}_{2, T}^{u *} & \boldsymbol{\Pi}_{1, T}^{u *}
\end{array}\right)
$$

associated with the noise subspace ${ }^{2}$ of $\mathbf{R}_{\tilde{y}, T}$ and $\Gamma_{\tilde{y}, T}^{u}$, respectively.

The following table summarizes the different models and algorithms that are defined in this paper:

\begin{tabular}{|c|c|c|c|}
\hline \multicolumn{2}{|c|}{ Standard data model } & \multicolumn{2}{c|}{ Robust distribution model } \\
\hline \multicolumn{2}{|c|}{ Circular noise: C-CG or C-CES } & C (or NC)-CES distributed observations $\mathbf{y}_{t}$ with scatter matrix: \\
\hline arbitrary sources & rectilinear sources & C-CES: $\mathbf{R}_{y}=\mathbf{A} \mathbf{R}_{x} \mathbf{A}^{H}+\sigma_{n}^{2} \mathbf{I}$ & NC-CES: $\mathbf{R}_{\tilde{y}}=\widetilde{\mathbf{A}} \mathbf{R}_{s} \widetilde{\mathbf{A}}^{H}+\sigma_{n}^{2} \mathbf{I}$ \\
\hline conventional MUSIC algorithm & NC MUSIC algorithm & conventional MUSIC algorithm & NC MUSIC algorithm \\
\hline $\mathbf{R}_{y, T} \longmapsto \boldsymbol{\Pi}_{T}$ & $\mathbf{R}_{\tilde{y}, T} \longmapsto \widetilde{\mathbf{\Pi}}_{T}$ & $\widetilde{\Gamma}_{y, T}^{u} \longmapsto \boldsymbol{\Pi}_{T}^{u}$ & $\widetilde{\boldsymbol{\Gamma}}_{\tilde{y}, T}^{u} \longmapsto \widetilde{\mathbf{\Pi}}_{T}^{u}$ \\
\hline
\end{tabular}

Table.1. Different models and algorithms studied in this paper.

\section{E. Statistical distributions of the SCM and M-estimators of covariance matrices}

For deriving in Section III-C the resolving power of these MUSIC algorithms based on $\mathbf{R}_{y, T}$ and $\mathbf{R}_{\tilde{y}, T}$, we will need the covariance $\mathbf{R}_{r_{y}} \stackrel{\text { def }}{=} \mathrm{E}\left[\left(\operatorname{vec}\left(\mathbf{R}_{y, T}\right)-\operatorname{vec}\left(\mathbf{R}_{y}\right)\right)\left(\operatorname{vec}^{H}\left(\mathbf{R}_{y, T}\right)-\operatorname{vec}\left(\mathbf{R}_{y}\right)\right)\right]$ and $\mathbf{R}_{r_{\tilde{y}}} \stackrel{\text { def }}{=} \mathrm{E}\left[\left(\operatorname{vec}\left(\mathbf{R}_{\tilde{y}, T}\right)-\right.\right.$ $\left.\left.\operatorname{vec}\left(\mathbf{R}_{\tilde{y}}\right)\right)\left(\operatorname{vec}^{H}\left(\mathbf{R}_{\tilde{y}, T}\right)-\operatorname{vec}\left(\mathbf{R}_{\tilde{y}}\right)\right)\right]$ of these SCM. They are given in [31] and [40], respectively, by

$$
\begin{aligned}
& \mathbf{R}_{r_{y}}=\frac{1}{T}\left[\left(\mathbf{R}_{y}^{*} \otimes \mathbf{R}_{y}\right)+\mathbf{K}\left(\mathbf{C}_{y} \otimes \mathbf{C}_{y}^{*}\right)+\left(\mathbf{A}^{*} \otimes \mathbf{A}\right) \mathbf{Q}_{x}\left(\mathbf{A}^{T} \otimes \mathbf{A}^{H}\right)+\mathbf{Q}_{n}\right], \\
& \mathbf{R}_{r_{\tilde{y}}}=\frac{1}{T}\left[\left(\mathbf{R}_{\tilde{y}}^{*} \otimes \mathbf{R}_{\tilde{y}}\right)+\mathbf{K}\left(\mathbf{C}_{\tilde{y}} \otimes \mathbf{C}_{\tilde{y}}^{*}\right)+\left(\widetilde{\mathbf{A}}^{*} \otimes \widetilde{\mathbf{A}}\right) \mathbf{Q}_{s}\left(\widetilde{\mathbf{A}}^{T} \otimes \widetilde{\mathbf{A}}^{H}\right)+\mathbf{Q}_{\tilde{n}}\right],
\end{aligned}
$$

where $\mathbf{Q}_{x}, \mathbf{Q}_{s}, \mathbf{Q}_{n}$ and $\mathbf{Q}_{\tilde{n}}$ are the quadrivariance ${ }^{3}$ of $\mathbf{x}_{t}, \mathbf{s}_{t}, \mathbf{n}_{t}$ and $\widetilde{\mathbf{n}}_{t}$, respectively. For C-CES distributed noise $\mathbf{Q}_{n}$ and $\mathbf{Q}_{\tilde{n}}$ are given in [31] and [29], respectively by

$$
\begin{aligned}
& \mathbf{Q}_{n}=\sigma_{n}^{4}(\eta-1)\left[\mathbf{I}+\operatorname{vec}(\mathbf{I}) \operatorname{vec}^{T}(\mathbf{I})\right], \\
& \mathbf{Q}_{\tilde{n}}=\sigma_{n}^{4}(\eta-1)\left[(\mathbf{I} \otimes \mathbf{I})+\mathbf{K}(\mathbf{J} \otimes \mathbf{J})+\operatorname{vec}(\mathbf{I}) \operatorname{vec}^{T}(\mathbf{I})\right] .
\end{aligned}
$$

Similarly to the standard data model, we will need the covariance of $\Gamma_{y, T}^{u}$ and $\Gamma_{\tilde{y}, T}^{u}$ for deriving in Section III-C the resolving power of the MUSIC algorithms based on these $M$-estimators. But only their asymptotic distributions are available. Under these Maronna conditions, it has been proved for RES distributions [41] and for C-CES distributions in [28], [42], then extended to NC-CES distributions in [29], that the sequences $\sqrt{T}\left(\operatorname{vec}\left(\boldsymbol{\Gamma}_{y, T}^{u}\right)-\operatorname{vec}\left(\sigma_{u} \mathbf{R}_{y}\right)\right)$ and $\sqrt{T}\left(\operatorname{vec}\left(\boldsymbol{\Gamma}_{\tilde{y}, T}^{u}\right)-\operatorname{vec}\left(\sigma_{u} \mathbf{R}_{\tilde{y}}\right)\right)$ converge in distribution to the zero mean Gaussian distribution $\mathcal{N}\left(\mathbf{0}, \mathbf{R}_{\Gamma_{y}^{u}}, \mathbf{C}_{\Gamma_{y}^{u}}\right)$ and

\footnotetext{
${ }^{2}$ Similar to the projector matrix $\boldsymbol{\Pi}_{T}$ associated with $\mathbf{R}_{T}$ from its EVD, the projector matrices $\widetilde{\boldsymbol{\Pi}}_{T}$ and $\widetilde{\boldsymbol{\Pi}}_{T}^{u}$ are defined by the EVD of $\mathbf{R}_{\tilde{y}, T}$ and $\boldsymbol{\Gamma}_{\tilde{y}, T}^{u}$, respectively, by collecting the eigenvectors associated with their $2 N-2$ smallest eigenvalues.

${ }^{3}$ For example $\left[\mathbf{Q}_{x}\right]_{i+2(j-1), k+2(l-1)}=\kappa_{i, j, k, l} \stackrel{\text { def }}{=} \operatorname{Cum}\left(x_{t, j}^{*}, x_{t, i}, x_{t, l}, x_{t, k}^{*}\right), i, j, k, l \in\{1,2\}$.
} 
$\mathcal{N}\left(\mathbf{0}, \mathbf{R}_{\Gamma_{\tilde{y}}^{u}}, \mathbf{C}_{\Gamma_{\tilde{y}}^{u}}\right)$, respectively, where

$$
\begin{aligned}
& \mathbf{R}_{\Gamma_{y}^{u}}=\vartheta_{1}\left(\mathbf{R}_{y}^{*} \otimes \mathbf{R}_{y}\right)+\vartheta_{2} \operatorname{vec}\left(\mathbf{R}_{y}\right) \operatorname{vec}^{H}\left(\mathbf{R}_{y}\right), \\
& \mathbf{R}_{\Gamma_{\tilde{y}}^{u}}=\vartheta_{1}\left(\left(\mathbf{R}_{\tilde{y}}^{*} \otimes \mathbf{R}_{\tilde{y}}\right)+\mathbf{K}\left(\mathbf{C}_{\tilde{y}} \otimes \mathbf{C}_{\tilde{y}}^{*}\right)\right)+\vartheta_{2} \operatorname{vec}\left(\mathbf{R}_{\tilde{y}}\right) \operatorname{vec}^{H}\left(\mathbf{R}_{\tilde{y}}\right)
\end{aligned}
$$

and with $\mathbf{C}_{\Gamma_{y}^{u}}=\mathbf{R}_{\Gamma_{y}^{u}} \mathbf{K}$ and $\mathbf{C}_{\Gamma_{\tilde{y}}^{u}}=\mathbf{R}_{\Gamma_{\tilde{y}}^{u}} \mathbf{K}$, where $\vartheta_{1}$ and $\vartheta_{2}$ are given by [28, (48-49)].

\section{RESOLVING POWER OF MUSIC-LIKE ALGORITHMS}

\section{A. Review on the resolving power}

Based upon the assumption [6] that the standard deviation $\sqrt{\operatorname{Var}\left[g_{T}^{\operatorname{Alg}_{\mathrm{C}}}(\theta)\right]}$ of the sample null spectrum associated with the conventional MUSIC and Min-Norm algorithms for circular Gaussian signals is small compared to its mean value $\mathrm{E}\left[g_{T}^{\mathrm{Alg}}{ }_{\mathrm{C}}(\theta)\right]$ in the vicinity of the true DOAs, the mean value of the sample null spectrum can be reasonably taken as representative of the ensemble of sample null spectra. We note that this assumption has been specified in [43], for which its validity has been proved in the conditions $N \gg 1$ and $T \gg N$ for the conventional MUSIC algorithm. We suppose it is also valid for non-Gaussian signals and as well as for the NC MUSIC algorithm. Based on this assumption, we continue to use the Cox [4] and the Sharman and Durrani [5] criteria which respectively state that two closely spaced equal-power sources are resolved if the following respective conditions are satisfied:

$$
\begin{aligned}
\mathrm{E}\left[g_{T}^{\mathrm{Alg}}\left(\theta_{1}\right)\right]=\mathrm{E}\left[g_{T}^{\mathrm{Alg}}\left(\theta_{2}\right)\right] & \leq \mathrm{E}\left[g_{T}^{\mathrm{Alg}}\left(\theta_{m}\right)\right] \\
\frac{d^{2} \mathrm{E}\left[g_{T}^{\mathrm{Alg}}(\theta)\right]}{d \theta^{2}} \mid \theta=\theta_{m} & \leq 0, \text { for } \theta_{m} \stackrel{\text { def }}{=}\left(\theta_{1}+\theta_{2}\right) / 2 .
\end{aligned}
$$

\section{B. Preliminary lemmas}

Approximations to the resolution threshold are deduced from equalities in (22) and (23). Consequently, the key point to derive these resolution thresholds depends on the expectation of the random variables $g_{T}^{\operatorname{Alg}_{\mathrm{C}}}(\theta)$ and $g_{T}^{\operatorname{Alg}_{\mathrm{NC}}}(\theta)$. To obtain these expectations, we resort to an analysis based on perturbations of the noise projector [38] instead of those of the eigenvectors (e.g., [6], [10]). Therefore, we consider the following second-order expansion of $\delta \boldsymbol{\Pi}_{T} \stackrel{\text { def }}{=} \Pi_{T}-\boldsymbol{\Pi}$ (where w.r.t. $\Pi$ denotes the projector matrix associated with the noise subspace of $\mathbf{R}_{y}$ ) w.r.t. $\delta \mathbf{R}_{y, T} \stackrel{\text { def }}{=} \mathbf{R}_{y, T}-\mathbf{R}_{y}$ proved in [38]:

$$
\begin{aligned}
\delta \boldsymbol{\Pi}_{T} & =-\left(\boldsymbol{\Pi} \delta \mathbf{R}_{y, T} \mathbf{S}^{\#}+\mathbf{S}^{\#} \delta \mathbf{R}_{y, T} \boldsymbol{\Pi}\right) \\
& +\mathbf{S}^{\#}\left(\delta \mathbf{R}_{y, T} \boldsymbol{\Pi} \delta \mathbf{R}_{y, T}\right) \mathbf{S}^{\#}-\mathbf{\Pi}\left(\delta \mathbf{R}_{y, T} \mathbf{S}^{\# 2} \delta \mathbf{R}_{y, T}\right) \boldsymbol{\Pi}+\mathbf{S}^{\#}\left(\delta \mathbf{R}_{y, T} \mathbf{S}^{\#} \delta \mathbf{R}_{y, T}\right) \boldsymbol{\Pi} \\
& +\boldsymbol{\Pi}\left(\delta \mathbf{R}_{y, T} \mathbf{S}^{\#} \delta \mathbf{R}_{y, T}\right) \mathbf{S}^{\#}-\mathbf{S}^{\# 2}\left(\delta \mathbf{R}_{y, T} \boldsymbol{\Pi} \delta \mathbf{R}_{y, T}\right) \boldsymbol{\Pi}-\boldsymbol{\Pi}\left(\delta \mathbf{R}_{y, T} \boldsymbol{\Pi} \delta \mathbf{R}_{y, T}\right) \mathbf{S}^{\# 2}+o\left(\delta \mathbf{R}_{y, T}^{2}\right)
\end{aligned}
$$

This relation is also valid for the extended model (6) by replacing $\boldsymbol{\Pi}, \boldsymbol{\Pi}_{T}, \delta \boldsymbol{\Pi}_{T}, \delta \mathbf{R}_{y, T}$ and $\mathbf{S}$, by $\widetilde{\boldsymbol{\Pi}}, \widetilde{\boldsymbol{\Pi}}_{T}, \delta \widetilde{\boldsymbol{\Pi}}_{T}, \delta \mathbf{R}_{\tilde{y}, T}$ and $\widetilde{\mathbf{S}}$, respectively. It is also valid for the robust distribution model, by replacing $\left(\delta \mathbf{\Pi}_{T}, \delta \mathbf{R}_{y, T}\right)$ and $\left(\delta \widetilde{\mathbf{\Pi}}_{T}, \delta \mathbf{R}_{\tilde{y}, T}\right)$, by $\left(\delta \boldsymbol{\Pi}_{T}^{u}, \delta \mathbf{R}_{y, T}^{u} \stackrel{\text { def }}{=} \sigma_{u}^{-1} \delta \boldsymbol{\Gamma}_{y, T}^{u}\right)$ and $\left(\delta \widetilde{\boldsymbol{\Pi}}_{T}^{u}, \delta \mathbf{R}_{\tilde{y}, T}^{u} \stackrel{\text { def }}{=} \sigma_{u}^{-1} \delta \boldsymbol{\Gamma}_{\tilde{y}, T}^{u}\right)$, respectively. 
To proceed, we need the expressions of $\mathrm{E}\left(\delta \mathbf{R}_{y, T} \mathbf{B}_{1} \delta \mathbf{R}_{y, T}\right)$ and $\mathrm{E}\left(\delta \mathbf{R}_{\tilde{y}, T} \mathbf{B}_{2} \delta \mathbf{R}_{\tilde{y}, T}\right)$ for arbitrary $N \times N$ matrices $\mathbf{B}_{1}$ and $2 N \times 2 N$ matrices $\mathbf{B}_{2}$, which are given by the following lemma proved in Appendix A:

Lemma 1: For the standard data model, we have

$$
\begin{aligned}
\mathrm{E}\left(\delta \mathbf{R}_{y, T} \mathbf{B}_{1} \delta \mathbf{R}_{y, T}\right) & =\frac{1}{T}\left(\operatorname{Tr}\left(\mathbf{B}_{1} \mathbf{R}_{y}\right) \mathbf{R}_{y}+\mathbf{C}_{y} \mathbf{B}_{1}^{T} \mathbf{C}_{y}^{*}+\sum_{i, j, k, l \in\{1,2\}} \kappa_{i, j, k, l} \mathbf{A} \mathbf{e}_{j} \mathbf{e}_{k}^{T} \mathbf{A}^{H} \mathbf{B}_{1} \mathbf{A} \mathbf{e}_{l} \mathbf{e}_{i}^{T} \mathbf{A}^{H}\right. \\
& \left.+\sigma_{n}^{4}(\eta-1)\left[\mathbf{B}_{1}+\operatorname{Tr}\left(\mathbf{B}_{1}\right) \mathbf{I}\right]\right), \\
\mathrm{E}\left(\delta \mathbf{R}_{\tilde{y}, T} \mathbf{B}_{2} \delta \mathbf{R}_{\tilde{y}, T}\right) & =\frac{1}{T}\left(\operatorname{Tr}\left(\mathbf{B}_{2} \mathbf{R}_{\tilde{y}}\right) \mathbf{R}_{\tilde{y}}+\mathbf{C}_{\tilde{y}} \mathbf{B}_{2}^{T} \mathbf{C}_{\tilde{y}}^{*}+\sum_{i, j, k, l \in\{1,2\}} \kappa_{i, j, k, l} \widetilde{\mathbf{A}} \mathbf{e}_{j} \mathbf{e}_{k}^{T} \widetilde{\mathbf{A}}^{H} \mathbf{B}_{2} \widetilde{\mathbf{A}} \mathbf{e}_{l} \mathbf{e}_{i}^{T} \widetilde{\mathbf{A}}^{H}\right. \\
& \left.+\sigma_{n}^{4}(\eta-1)\left[\mathbf{B}_{2}+\mathbf{J} \mathbf{B}_{2}^{T} \mathbf{J}+\operatorname{Tr}\left(\mathbf{B}_{2}\right) \mathbf{I}\right]\right),
\end{aligned}
$$

and for the robust distribution model:

$$
\begin{aligned}
\mathrm{E}\left(\delta \mathbf{R}_{y, T}^{u} \mathbf{B}_{1} \delta \mathbf{R}_{y, T}^{u}\right) & \approx \frac{1}{T \sigma_{u}^{2}}\left(\vartheta_{1} \operatorname{Tr}\left(\mathbf{B}_{1} \mathbf{R}_{y}\right) \mathbf{R}_{y}+\vartheta_{2} \mathbf{R}_{y} \mathbf{B}_{1}^{T} \mathbf{R}_{y}\right) \\
\mathrm{E}\left(\delta \mathbf{R}_{\tilde{y}, T}^{u} \mathbf{B}_{2} \delta \mathbf{R}_{\tilde{y}, T}^{u}\right) & \approx \frac{1}{T \sigma_{u}^{2}}\left(\vartheta_{1} \operatorname{Tr}\left(\mathbf{B}_{2} \mathbf{R}_{\tilde{y}}\right) \mathbf{R}_{\tilde{y}}+\vartheta_{1} \mathbf{C}_{\tilde{y}} \mathbf{B}_{2}^{T} \mathbf{C}_{\tilde{y}}^{*}+\vartheta_{2} \mathbf{R}_{\tilde{y}} \mathbf{B}_{2} \mathbf{R}_{\tilde{y}}\right) .
\end{aligned}
$$

Then using Lemma 1 with $\mathbf{B}_{1}=\mathbf{\Pi}, \mathbf{S}^{\#}$ and $\mathbf{S}^{\# 2}$, then with $\mathbf{B}_{2}=\widetilde{\mathbf{\Pi}}, \widetilde{\mathbf{S}} \#$ and $\widetilde{\mathbf{S}}^{\# 2}$ in the derivation of the mean of $\delta \boldsymbol{\Pi}_{T}$ given in (24) and the mean of $\delta \widetilde{\boldsymbol{\Pi}}_{T}$, we get after some algebraic manipulations based on the identities $\mathbf{R}_{y} \Pi=\sigma_{n}^{2} \boldsymbol{\Pi}$, $\mathbf{\Pi C}_{y}=\mathbf{0}, \mathbf{S}^{\#} \boldsymbol{\Pi}=\mathbf{0}$ and $\mathbf{R}_{\tilde{y}} \widetilde{\boldsymbol{\Pi}}=\sigma_{n}^{2} \widetilde{\boldsymbol{\Pi}}, \mathbf{J} \widetilde{\boldsymbol{\Pi}}^{T} \mathbf{J}=\tilde{\mathbf{\Pi}}, \mathbf{J} \widetilde{\mathbf{S}} \#^{T} \mathbf{J}=\widetilde{\mathbf{S}} \#$ and $\mathbf{J} \widetilde{\mathbf{S}} \# 2^{T} \mathbf{J}=\widetilde{\mathbf{S}}{ }^{2}$, the following lemma:

Lemma 2: In the standard data and robust distribution models, the mean of $\delta \boldsymbol{\Pi}_{T}$ and $\delta \boldsymbol{\Pi}_{T}^{u}$ are given respectively by

$$
\begin{gathered}
\mathrm{E}\left(\delta \mathbf{\Pi}_{T}\right) \approx \frac{1}{T}\left(\operatorname{Tr}(\boldsymbol{\Pi}) \mathbf{U}-\operatorname{Tr}(\mathbf{U}) \boldsymbol{\Pi}+(\eta-1)\left(\operatorname{Tr}(\mathbf{\Pi}) \mathbf{U}^{\prime}-\operatorname{Tr}\left(\mathbf{U}^{\prime}\right) \boldsymbol{\Pi}\right)\right), \\
\mathrm{E}\left(\delta \boldsymbol{\Pi}_{T}^{u}\right) \approx \frac{\vartheta_{1}}{T \sigma_{u}^{2}}(\operatorname{Tr}(\boldsymbol{\Pi}) \mathbf{U}-\operatorname{Tr}(\mathbf{U}) \boldsymbol{\Pi}),
\end{gathered}
$$

with $\mathbf{U} \stackrel{\text { def }}{=} \sigma_{n}^{2} \mathbf{S}^{\#} \mathbf{R}_{y} \mathbf{S}^{\#}$ and $\mathbf{U}^{\prime} \stackrel{\text { def }}{=} \sigma_{n}^{4} \mathbf{S}^{\# 2}$.

For the two extended models, the mean of $\delta \widetilde{\boldsymbol{\Pi}}_{1, T}, \delta \widetilde{\boldsymbol{\Pi}}_{2, T}, \delta \widetilde{\mathbf{\Pi}}_{1, T}^{u}$ and $\delta \widetilde{\boldsymbol{\Pi}}_{2, T}^{u}$ are given respectively by

$$
\begin{aligned}
& \mathrm{E}\left(\delta \boldsymbol{\Pi}_{1, T}\right) \approx \frac{2}{T}\left(\operatorname{Tr}\left(\boldsymbol{\Pi}_{1}\right) \mathbf{U}_{1}-\operatorname{Tr}\left(\mathbf{U}_{1}\right) \boldsymbol{\Pi}_{1}+(\eta-1)\left(\operatorname{Tr}\left(\boldsymbol{\Pi}_{1}\right) \mathbf{U}_{1}^{\prime}-\operatorname{Tr}\left(\mathbf{U}_{1}^{\prime}\right) \boldsymbol{\Pi}_{1}\right)\right) \\
& \mathrm{E}\left(\delta \boldsymbol{\Pi}_{2, T}\right) \approx \frac{2}{T}\left(\operatorname{Tr}\left(\boldsymbol{\Pi}_{1}\right) \mathbf{U}_{2}-\operatorname{Tr}\left(\mathbf{U}_{1}\right) \boldsymbol{\Pi}_{2}+(\eta-1)\left(\operatorname{Tr}\left(\boldsymbol{\Pi}_{1}\right) \mathbf{U}_{2}^{\prime}-\operatorname{Tr}\left(\mathbf{U}_{1}^{\prime}\right) \boldsymbol{\Pi}_{2}\right)\right) \\
& \mathrm{E}\left(\delta \boldsymbol{\Pi}_{1, T}^{u}\right) \approx \frac{2 \vartheta_{1}}{T \sigma_{u}^{2}}\left(\operatorname{Tr}\left(\boldsymbol{\Pi}_{1}\right) \mathbf{U}_{1}-\operatorname{Tr}\left(\mathbf{U}_{1}\right) \boldsymbol{\Pi}_{1}\right) \\
& \mathrm{E}\left(\delta \mathbf{\Pi}_{2, T}^{u}\right) \approx \frac{2 \vartheta_{1}}{T \sigma_{u}^{2}}\left(\operatorname{Tr}\left(\boldsymbol{\Pi}_{1}\right) \mathbf{U}_{2}-\operatorname{Tr}\left(\mathbf{U}_{1}\right) \boldsymbol{\Pi}_{2}\right)
\end{aligned}
$$

where $\mathbf{U}_{1}$ and $\mathbf{U}_{2}, \mathbf{U}_{1}^{\prime}$ and $\mathbf{U}_{2}^{\prime}, \Pi_{1}$ and $\Pi_{2}$ are $N \times N$ sub-block matrices of the $2 N \times 2 N$ Hermitian positive semidefinite matrices $\widetilde{\mathbf{U}} \stackrel{\text { def }}{=} \sigma_{n}^{2} \widetilde{\mathbf{S}}^{\#} \mathbf{R}_{\tilde{y}} \widetilde{\mathbf{S}}^{\#}=\left(\begin{array}{cc}\mathbf{U}_{1} & \mathbf{U}_{2} \\ \mathbf{U}_{2}^{*} & \mathbf{U}_{1}^{*}\end{array}\right), \widetilde{\mathbf{U}}^{\prime} \stackrel{\text { def }}{=} \sigma_{n}^{4} \widetilde{\mathbf{S}}^{\# 2}=\left(\begin{array}{cc}\mathbf{U}_{1}^{\prime} & \mathbf{U}_{2}^{\prime} \\ \mathbf{U}_{2}^{\prime *} & \mathbf{U}_{1}^{\prime *}\end{array}\right)$ and $\widetilde{\boldsymbol{\Pi}}=\left(\begin{array}{cc}\boldsymbol{\Pi}_{1} & \boldsymbol{\Pi}_{2} \\ \boldsymbol{\Pi}_{2}^{*} & \boldsymbol{\Pi}_{1}^{*}\end{array}\right)$, respectively. Using (29) and (30) in (13), allows us to straightforward derive the mean null spectra associated with the conventional 
MUSIC algorithm based on the SCM, then on an $M$-estimate covariance of $\mathbf{y}_{t}$, which are given respectively by:

$$
\begin{aligned}
\mathrm{E}\left(g_{T}^{\operatorname{Alg}_{\mathrm{C}}}(\theta)\right) & \approx g^{\mathrm{Alg}}(\theta)+\frac{1}{T}\left((N-2) \mathbf{a}^{H}(\theta) \mathbf{U} \mathbf{a}(\theta)-\operatorname{Tr}(\mathbf{U}) g^{\operatorname{Alg}_{\mathrm{C}}}(\theta)\right. \\
& \left.+(\eta-1)\left((N-2) \mathbf{a}^{H}(\theta) \mathbf{U}^{\prime} \mathbf{a}(\theta)-\operatorname{Tr}\left(\mathbf{U}^{\prime}\right) g^{\operatorname{Alg} \lg _{\mathrm{C}}}(\theta)\right)\right) \\
\mathrm{E}\left(g_{T}^{\operatorname{Alg}_{\mathrm{C}}^{\mathrm{u}}}(\theta)\right) & \approx g^{\operatorname{Alg}_{\mathrm{C}}}(\theta)+\frac{\vartheta_{1}}{T \sigma_{u}^{2}}\left((N-2) \mathbf{a}^{H}(\theta) \mathbf{U} \mathbf{a}(\theta)-\operatorname{Tr}(\mathbf{U}) g^{\operatorname{Alg}_{\mathrm{C}}}(\theta)\right),
\end{aligned}
$$

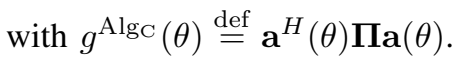

For the NC MUSIC algorithm, the derivation of the mean null spectra is more involved, but using asymptotic distribution of $\boldsymbol{\Pi}_{1, T}, \boldsymbol{\Pi}_{2, T}, \boldsymbol{\Pi}_{1, T}^{u}$ and $\boldsymbol{\Pi}_{2, T}^{u}$ given in [29], the mean null spectra for this algorithm based on the SCM and then on an $M$-estimate covariance of $\tilde{\mathbf{y}}_{t}$ are proved in Appendix B and given respectively by:

$$
\begin{aligned}
& \mathrm{E}\left(g_{T}^{\operatorname{Alg}_{\mathrm{NC}}}(\theta)\right) \approx g^{\operatorname{Alg}_{\mathrm{NC}}}(\theta) \\
& +\frac{2}{T}(2 N-3)\left[\left(\mathbf{a}^{H}(\theta) \mathbf{U}_{1} \mathbf{a}(\theta)\right)\left(\mathbf{a}^{H}(\theta) \boldsymbol{\Pi}_{1} \mathbf{a}(\theta)\right)-\operatorname{Re}\left[\left(\mathbf{a}^{H}(\theta) \mathbf{U}_{2} \mathbf{a}^{*}(\theta)\right)\left(\mathbf{a}^{T}(\theta) \boldsymbol{\Pi}_{2}^{*} \mathbf{a}(\theta)\right)\right]\right] \\
& +\frac{2(\eta-1)}{T}(2 N-3)\left[\left(\mathbf{a}^{H}(\theta) \mathbf{U}_{1}^{\prime} \mathbf{a}(\theta)\right)\left(\mathbf{a}^{H}(\theta) \boldsymbol{\Pi}_{1} \mathbf{a}(\theta)\right)-\operatorname{Re}\left[\left(\mathbf{a}^{H}(\theta) \mathbf{U}_{2}^{\prime} \mathbf{a}^{*}(\theta)\right)\left(\mathbf{a}^{T}(\theta) \boldsymbol{\Pi}_{2}^{*} \mathbf{a}(\theta)\right)\right]\right] \\
& -\frac{4}{T}\left[\operatorname{Tr}\left(\mathbf{U}_{1}\right)+(\eta-1) \operatorname{Tr}\left(\mathbf{U}_{1}^{\prime}\right)\right] g^{\operatorname{Alg}_{\mathrm{NC}}}(\theta) \\
& \mathrm{E}\left(g_{T}^{\mathrm{Alg}_{\mathrm{NC}}^{\mathrm{u}}}(\theta)\right) \approx g^{\mathrm{Alg} \mathrm{NC}}(\theta) \\
& +\frac{2 \vartheta_{1}}{T \sigma_{u}^{2}}(2 N-3)\left[\left(\mathbf{a}^{H}(\theta) \mathbf{U}_{1} \mathbf{a}(\theta)\right)\left(\mathbf{a}^{H}(\theta) \boldsymbol{\Pi}_{1} \mathbf{a}(\theta)\right)-\operatorname{Re}\left[\left(\mathbf{a}^{H}(\theta) \mathbf{U}_{2} \mathbf{a}^{*}(\theta)\right)\left(\mathbf{a}^{T}(\theta) \boldsymbol{\Pi}_{2}^{*} \mathbf{a}(\theta)\right)\right]\right] \\
& -\frac{4 \vartheta_{1}}{T \sigma_{u}^{2}} \operatorname{Tr}\left(\mathbf{U}_{1}\right) g^{\operatorname{Alg}_{N C}}(\theta)
\end{aligned}
$$

with $g^{\operatorname{Alg} g_{N C}}(\theta) \stackrel{\text { def }}{=}\left(\mathbf{a}^{H}(\theta) \boldsymbol{\Pi}_{1} \mathbf{a}(\theta)\right)^{2}-\left|\mathbf{a}^{T}(\theta) \boldsymbol{\Pi}_{2}^{*} \mathbf{a}(\theta)\right|^{2}$.

We check that (35) and (37) reduce to [13, rel. (3.6)] and [13, rel. (4.4)], respectively, for circular Gaussian noise for which $\eta=1$. Likewise, (36) and (38) reduce to [13, rel. (3.6)] and [13, rel. (4.4)], respectively, for Gaussian observations associated with the SCM for which $\sigma_{u}=\vartheta_{1}=1$.

Finally, note that all the results of this subsection apply to an arbitrary number $K$ of sources necessarily equi-powered by by replacing $N-2$ by $N-K$ in (35) (36) and $2 N-3$ by $2 N-K-1$ in (37) (38).

\section{Derivation of the threshold ASNR}

First, we note that mean null spectra associated with the standard data model (35) and (37) reduce to those of the robust distribution model (36) and (38) if $\eta=1$ and $T$ replaced by $T \sigma_{u}^{2} / \vartheta_{1}$. Consequently, the threshold ASNR for the robust distribution model is directly deduced form those of the standard data model. Let us start with the derivation of the threshold ASNR given by the Cox (22) and the Sharman and Durrani (23) criteria, applied to the conventional MUSIC algorithm for the standard data model. To obtain these threshold ASNRs, we must get closed-form expressions of $\mathbf{a}^{H}(\theta) \mathbf{U a}(\theta)$, $\mathbf{a}^{H}(\theta) \mathbf{U}^{\prime} \mathbf{a}(\theta)$ and $\mathbf{a}^{H}(\theta) \Pi \mathbf{a}(\theta)$ in (35). To simplify notations and to make the calculations of these expressions easier, we derive in Appendix $C$, the matrices $\Pi, \mathbf{U}$ and $\mathbf{U}^{\prime}$ for only centrosymmetric arrays where the coordinate system has its 
origin at the centroid of the arrays ${ }^{4}$. Plugging their expressions in (35) and using symbolic calculus akin to a high level language, the terms $\mathrm{E}\left(g_{T}^{\operatorname{Alg} \mathrm{C}}\left(\theta_{1}\right)\right), \mathrm{E}\left(g_{T}^{\operatorname{Alg}_{\mathrm{C}}}\left(\theta_{m}\right)\right)$ and $\frac{d^{2} \mathrm{E}\left[g_{T}^{\mathrm{Alg}_{\mathrm{C}}}(\theta)\right]}{d \theta^{2}} \mid \theta=\theta_{m}$ of the Cox and the Sharman and Durrani criteria, can be written in the following form:

$$
\begin{aligned}
\mathrm{E}\left(g_{T}^{\mathrm{Alg}_{\mathrm{C}}}\left(\theta_{1}\right)\right) & \approx \frac{1}{T}\left[\frac{1}{r} h_{1,1}+\frac{1}{r^{2}} h_{2,1}\right] \\
\mathrm{E}\left(g_{T}^{\mathrm{Alg}_{\mathrm{C}}}\left(\theta_{m}\right)\right) & \approx g^{\mathrm{Alg} \mathrm{C}}\left(\theta_{m}\right)+\frac{1}{T}\left[\frac{1}{r} h_{1, m}+\frac{1}{r^{2}} h_{2, m}\right] \\
\frac{d^{2} \mathrm{E}\left[g_{T}^{\mathrm{Alg}_{\mathrm{C}}}(\theta)\right]}{d \theta^{2}} \mid \theta=\theta_{m} & \approx \frac{d^{2} g^{\mathrm{Alg}}(\theta)}{d \theta^{2}} \mid \theta=\theta_{m}
\end{aligned}
$$

where $r \stackrel{\text { def }}{=} N \sigma_{x}^{2} / \sigma_{n}^{2}$ and the terms $h_{1,1}, h_{2,1}, h_{1, m}, h_{2, m} h_{1, m}^{\prime}$ and $h_{2, m}^{\prime}$ given in Appendix D for arbitrary centrosymmetric arrays are functions of the real-valued geometric terms $\mathbf{a}_{1}^{H} \mathbf{a}_{2}, \mathbf{a}_{1}^{H} \mathbf{a}_{m}, \mathbf{a}_{m}^{\prime}{ }^{H} \mathbf{a}_{1}$ and $\mathbf{a}_{m}^{\prime \prime} \mathbf{a}_{1}$. Using expansions of these terms with respect to $\delta \theta \stackrel{\text { def }}{=} \theta_{2}-\theta_{1}$ according to the parameterization of [10] for closely-spaced sources allow us to prove the following result:

Result 1: The threshold ASNRs deduced from the Cox (22) and the Sharman and Durrani (23) criteria given by the conventional MUSIC algorithm (12) for both standard data (section II-B) and robust distribution (section II-C) models with two correlated equal-power sources and an arbitrary centrosymmetric array are given for closely-spaced sources and a large number $T$ of snapshots, by the following approximations:

$$
\xi_{\mathrm{C}-\mathrm{MUSIC}} \approx \frac{\delta_{1} \delta_{2}}{T}\left(\frac{1+\operatorname{Re}(\rho)}{1-|\rho|^{2}}\right) \frac{\alpha_{N}}{(\Delta \theta)^{4}}\left(1+\sqrt{1+\frac{\delta_{3} T}{\delta_{1} \delta_{2}} \frac{(\Delta \theta)^{2}}{\beta_{N}}}\right),
$$

where $\Delta \theta$ denotes the measure ${ }^{5}$ of the angular separation between the two sources defined by [10, rel. (8)] and where the parameters $\delta_{1}, \delta_{2}$ and $\delta_{3}$ are defined in Table 2 .

\begin{tabular}{|c|c|}
\hline Cox criteria & $\delta_{1}=2$ \\
\hline Sherman and Durrani criteria & $\delta_{1}=1$ \\
\hline Standard data model & $\left(\delta_{2}, \delta_{3}\right)=(1, \eta)$ \\
\hline Robust distribution model & $\left(\delta_{2}, \delta_{3}\right)=\left(\vartheta_{1} / \sigma_{u}^{2}, 1\right)$ \\
\hline
\end{tabular}

Table 2. Parameters $\delta_{1}, \delta_{2}$ and $\delta_{3}$

In the specific case of ULA associated with the symmetric steering vectors

$$
\mathbf{a}(\theta)=\left(e^{-i \frac{(N-1) \theta}{2}}, e^{-i \frac{(N-3) \theta}{2}}, . ., e^{i \frac{(N-3) \theta}{2}}, e^{i \frac{(N-1) \theta}{2}}\right)^{T}
$$

${ }^{4}$ We note that this structure is very used in practice because uniform linear, uniform circular and regular hexagonal shaped arrays [44], cross-based centro-symmetric arrays, square-based centro-symmetric array are centrosymmetric.

${ }^{5}$ This measure is defined by $(\Delta \theta)^{2} \stackrel{\text { def }}{=} \frac{(2 \pi)^{2}}{\lambda^{2} N^{2}} \sum_{n=1}^{N}\left[\mathbf{r}_{n}^{T}\left(\mathbf{s}_{1}-\mathbf{s}_{2}\right)\right]^{2}$, where $\mathbf{r}_{n}, \mathbf{s}_{1}$ and $\mathbf{s}_{2}$ denote vectors pointing from the centroid of the array to the $n$-th sensor and to the two sources, and $\lambda$ is the wavelength. 
where the coordinate system has its origin at the centroid of the array, this measure of angular separation $\Delta \theta$ for closelyspaced sources is given by

$$
\Delta \theta \approx N \delta \theta \cos \theta_{m} /(2 \sqrt{3}) \text { where } \theta_{m} \stackrel{\text { def }}{=} \frac{1}{2}\left(\theta_{1}+\theta_{2}\right)
$$

and $\alpha_{N}$ and $\beta_{N}$ are given by:

$$
\alpha_{N}=\frac{10 N^{4} \cos ^{4} \theta_{m}}{\left(N^{2}-1\right)(N+2)} \text { and } \beta_{N}=\frac{5 N^{2} \cos ^{2} \theta_{m}}{2(N+2)} .
$$

For the NC MUSIC algorithm, the derivation of the threshold ASNRs given by the Cox and the Sharman and Durrani criteria follows the same steps. Closed-form expressions of the blocks $\Pi_{1}, \Pi_{2}, \mathbf{U}_{1}, \mathbf{U}_{2}$ and $\mathbf{U}_{1}^{\prime}, \mathbf{U}_{2}^{\prime}$ of $\widetilde{\Pi}, \widetilde{\mathbf{U}}$ and $\widetilde{\mathbf{U}}^{\prime}$, respectively, are derived in Appendix D and E. Note that the expression $\left[\mathbf{a}_{k}^{T} e^{i \phi_{k}}, \mathbf{a}_{k}^{H} e^{-i \phi_{k}}\right]^{T}$ of the extended steering vectors allows for simpler calculations than for the expression $\left[\mathbf{a}_{k}^{T}, \mathbf{a}_{k}^{H} e^{-i 2 \phi_{k}}\right]^{T}$ used in the literature. Using symbolic calculus, (39), (40) and (41) can also be derived for the mean spectrum $\mathrm{E}\left[g_{T}^{\mathrm{Alg}_{\mathrm{NC}}}(\theta)\right]$ where now $h_{1,1}, h_{2,1}, h_{1, m}, h_{2, m} h_{1, m}^{\prime}$ and $h_{2, m}^{\prime}$ are functions of both $\Delta \theta$ and $\Delta \phi$. Using expansion of these spatial terms with respect to $\Delta \theta$ for closely-spaced sources allow us to prove the following result:

Result 2: The threshold ASNRs deduced from the Cox (22) and the Sharman and Durrani (23) criteria given by the NC MUSIC algorithm (14) for both standard data (section II-B) and robust distribution (section II-C) models with two correlated equal-power sources and an arbitrary centrosymmetric array are respectively given for closely-spaced sources and a large number $T$ of snapshots, by the following approximations:

$$
\xi_{\text {NC-MUSIC }} \approx \frac{\delta_{1} \delta_{2}}{T}\left(\frac{1+\rho^{\prime} \cos (\Delta \phi)}{\left(1-\rho^{\prime 2}\right) \sin ^{2}(\Delta \phi)}\right) \frac{\gamma_{N}}{(\Delta \theta)^{2}}\left(1+\sqrt{1+\frac{\delta_{3} T}{\delta_{1} \delta_{2}} \frac{(\Delta \theta)^{2}}{\gamma_{N}}\left[1+\left(\frac{\rho^{\prime}+\cos (\Delta \phi)}{1+\rho^{\prime} \cos (\Delta \phi)}\right)^{2}\right]}\right),
$$

except for "very small" $\Delta \phi \bmod \pi$ for which:

$$
\xi_{\mathrm{NC}-\mathrm{MUSIC}} \approx \frac{\delta_{1} \delta_{2}}{T}\left(\frac{1}{1-\rho^{\prime}}\right) \frac{\alpha_{N}}{(\Delta \theta)^{4}}\left(1+\sqrt{1+\frac{\delta_{3} T}{\delta_{1} \delta_{2}} \frac{(\Delta \theta)^{2}}{\beta_{N}}}\right)
$$

where $\delta_{1}, \delta_{2}$ and $\delta_{3}$ are defined in Table 2 .

In the specific case of ULA whose steering vector given by (43) and where $\Delta \theta$ is defined by (44), we have

$$
\gamma_{N}=\frac{N^{2}(2 N-3) \cos ^{2} \theta_{m}}{N^{2}-1}, \alpha_{N}=\frac{5 N^{4}(2 N-3) \cos ^{4} \theta_{m}}{\left(N^{2}-1\right)\left(N^{2}-4\right)} \text { and } \beta_{N}=\frac{5 N^{2}(2 N-3) \cos ^{2} \theta_{m}}{N^{2}-4} .
$$

We note that for Gaussian distributed noise in the standard data model for which $\eta=1$ and Gaussian distributed observations in the robust distribution model for which $\vartheta_{1}=\sigma_{u}=1$, we have $\delta_{2}=\delta_{3}=1$ in (42), (45) and (46), In this case, for uncorrelated sources $\left(\rho=\rho^{\prime}=0\right)$, the threshold ASNRs (42), (45) and (46) reduce to [13, (3.7)(3.8)], $[13,(4.5)(4.6)(4.8)]$ and $[13,(4.5)(4.6)(4.7)]$, respectively.

\section{General comments}

This section attempts to explain the influence of the signal and noise parameters on the ASNR thresholds in Results 1 and 2 . 
1) Influence of the non-Gaussianity of the noise and observations: In the standard data model for which $\delta_{2}=1$ and $\delta_{3}=\eta$, the non-Gaussianity of the noise can strongly impact the threshold ASNR. This is particularly the case of the C-CCG noise distributions [28] including the circular complex Student $t$ and generalized Gaussian distributions, for which $\eta>1$ where $\eta$ is very large for heavy-tailed distributions. Thus for these distributions, the required ASNR to resolve closely-spaced sources can be very large, as it will be illustrated in Section IV.

In the robust distribution model for which $\delta_{2}=\vartheta_{1} / \sigma_{u}^{2}$ and $\delta_{3}=1$, the non-Gaussianity of the observation impacts the threshold ASNR through the equivalent number $T / \delta_{2}=\sigma_{u}^{2} T / \vartheta_{1}$ of snapshots. For ML estimates of $\mathbf{R}_{y}$ and $\mathbf{R}_{\tilde{y}}, \sigma_{u}=1$ and $\vartheta_{1}$ is close to 1 for many heavy-tailed CES distributions. Likewise, for the Tyler's $M$-estimate, $\vartheta_{1}=(N+1) / N$ [37]. Consequently, the threshold ASNRs (42), (45) and (46) required for heavy-tailed CES distributions are very similar to those obtained in the case of Gaussian distributions. In contrast if the SCM have been used to estimate $\mathbf{R}_{y}$ and $\mathbf{R}_{\tilde{y}}$, $u(t)=1$ gives $\sigma_{u}=1$ and $\vartheta_{1}=\eta$ and the equivalent number $T / \delta_{2}=T / \eta$ of snapshots is dramatically reduced for heavy-tailed distributions and thus, the required ASNR to resolve closely-spaced sources would be very large.

2) Influence of the correlation (magnitude and phase) for the conventional MUSIC algorithm: The threshold ASNR (42) is a function of the magnitude of the correlation of sources, but also of its phase. Note that many performance analysis of the conventional MUSIC algorithm seem to have always assumed that the correlation phase is zero or $180^{\circ}$ (e.g., in [45]). Whereas, it is known [46] that the correlation phase has a strong effect on the associated Cramér-Rao bound under certain conditions (small aperture arrays, large correlation magnitude and closely-spaced sources).

As expected, the threshold ASNR generally increases strongly as the magnitude of the correlation approaches one for which the sources are coherent. In this case, the signal subspace is one-dimensional and the conventional MUSIC algorithm fails. More precisely, the relevant correlation term in (42) satisfies the following property:

$$
\begin{aligned}
\left.\frac{1+\operatorname{Re}(\rho)}{1-|\rho|^{2}}\right|_{\angle \rho \neq \pi} & =\left.\frac{1+|\rho| \cos (\angle \rho)}{1-|\rho|^{2}}\right|_{\angle \rho \neq \pi} \underset{|\rho| \rightarrow+1}{\longrightarrow}+\infty \\
\left.\frac{1+\operatorname{Re}(\rho)}{1-|\rho|^{2}}\right|_{\angle \rho=\pi} & =\frac{1}{1+|\rho|} \underset{|\rho| \rightarrow+1}{\longrightarrow} 1 / 2 .
\end{aligned}
$$

In this latter case the threshold ASNR reaches half the value for uncorrelated sources. This singular case occurs when the distance $\mathrm{E}\left|x_{t, 1}-x_{t, 2}\right|^{2}$ reaches its maximum.

However, for a fixed magnitude correlation, the phase correlation can also strongly impact the threshold ASNR. We can clearly observe that the threshold ASNR (42) is maximal [resp., minimal] for $\angle \rho=0$ [resp., $\angle \rho=\pi$ ] where the term $\frac{1+\operatorname{Re}(\rho)}{1-|\rho|^{2}}$ of (42) takes the value $\frac{1}{1-|\rho|}$ and $\frac{1}{1+|\rho|}$, respectively. We can deduce that for $\angle \rho=\pi$, two correlated sources are better resolved than uncorrelated sources with the conventional MUSIC algorithm.

3) Influence of the correlation and phase separation of rectilinear sources for the NC MUSIC algorithm: It can also be seen, comparing (45) to (42), that the NC MUSIC algorithm always largely outperforms the conventional MUSIC algorithm used for rectilinear sources, due to the proportionality of $\xi_{\mathrm{NC}-\mathrm{MUSIC}}$ and $\xi_{\mathrm{C}-\mathrm{MUSIC}}$ to $1 /(\Delta \theta)^{2}$ and $1 /(\Delta \theta)^{4}$, respectively.

In contrast, for very weak $\Delta \phi(\bmod \pi)$, the behavior of the conventional and NC MUSIC algorithms are similar due to the similarity of the dependence in $(\Delta \theta)^{4}$ in (42) and (46). The weak $\Delta \phi(\bmod \pi)$ correspond to the worst resolution 
capability of the NC MUSIC algorithm. Consequently, the phase separation $\Delta \phi$ plays a crucial role from the resolution point of view for any distribution of noise and observations.

For uncorrelated sources $\left(\rho^{\prime}=0\right)$, (45) clearly shows that the threshold ASNR deduced from the Cox and the Sharman and Durrani criteria given by the NC MUSIC algorithm is minimum for $\Delta \phi=\pi / 2$. Furthermore (45) shows that $\xi_{\mathrm{NC}-\mathrm{MUSIC}}$ is a symmetric function of $\Delta \phi$ with respect to $\pi / 2$. This property is consistent with the asymptotic variance of the NC MUSIC DOA estimation algorithm, which has been observed numerically minimal for $\Delta \phi=\pi / 2$ [47].

For correlated sources, the role of $\Delta \phi$ in (45) is more complicated to analyze. However, noting that for $\rho^{\prime}>0$, $\frac{1+\rho^{\prime} \cos (\Delta \phi)}{\left(1-\rho^{\prime 2}\right) \sin ^{2}(\Delta \phi)}$ and $\left(\frac{\rho^{\prime}+\cos (\Delta \phi)}{1+\rho^{\prime} \cos (\Delta \phi)}\right)^{2}$ are decreasing functions of $\Delta \phi$ in $[0, \pi]$ and $\left[0, \Delta \phi_{0}\right]$, respectively (where $\Delta \phi_{0}$ is solution of $\rho^{\prime}+\cos \left(\Delta \phi_{0}\right)=0$ ), the threshold ASNR (45) is minimum for $\Delta \phi=\Delta \phi_{1}>\Delta \phi_{0}>\pi / 2$, where $\Delta \phi_{1}$ increases as $\rho^{\prime}$ increases. With the same approach, for $\rho^{\prime}<0$, the threshold ASNR is minimum for $\Delta \phi=\Delta \phi_{1}<\Delta \phi_{0}<\pi / 2$, where $\Delta \phi_{1}$ decreases as $\rho^{\prime}$ decreases. This dependence of $\xi_{\mathrm{NC}-\mathrm{MUSIC}}$ to $\Delta \phi$ for different values of $\rho^{\prime}$ will be illustrated in Fig.5 in Section IV. Furthermore, we note that for $\Delta \phi=\pi / 2, \frac{1+\rho^{\prime} \cos (\Delta \phi)}{\left(1-\rho^{\prime 2}\right) \sin ^{2}(\Delta \phi)}=\frac{1}{1-\rho^{\prime 2}}$ and $\left(\frac{\rho^{\prime}+\cos (\Delta \phi)}{1+\rho^{\prime} \cos (\Delta \phi)}\right)^{2}=\rho^{\prime 2}$, and therefore the threshold ASNR does depend on the sign of $\rho^{\prime} \in(-1,+1)$.

Note also that the threshold ASNR associated with NC MUSIC algorithm generally increases strongly as the magnitude of the correlation approaches one because $\frac{1+\rho^{\prime} \cos (\Delta \phi)}{\left(1-\rho^{\prime 2}\right) \sin ^{2}(\Delta \phi)} \rightarrow+\infty$ for $\Delta \phi \neq 0$ and $\rho^{\prime} \rightarrow \pm 1$ in (45) and $\frac{1}{1-\rho^{\prime}} \rightarrow+\infty$ for $\Delta \phi=0$ and $\rho^{\prime} \rightarrow+1$ in (46). However, $\frac{1}{1-\rho^{\prime}} \rightarrow 1 / 2$ for $\Delta \phi=0$ and $\rho^{\prime} \rightarrow-1$ in (46), which is consistent with the singular case of the conventional MUSIC algorithm, whose performances are similar for $\Delta \phi=0$.

4) Fluctuation of the correlation phase: Finally note that the phase of the correlation $\rho$ of the sources for the conventional MUSIC algorithm (which corresponds to $\Delta \phi$ for rectilinear sources, see (9)) is a highly variable and unpredictable parameter in a multipath environment for which it is very sensitive to the difference between the propagation delays in the direct and secondary paths. Consequently, the threshold ASNRs may vary significantly from time to time and thus the resolution performance are rather given by the mean of the threshold ASNRs with respect to the correlation phase. For the conventional MUSIC algorithm, (42) gives:

$$
\mathrm{E}\left(\xi_{\mathrm{C}-\mathrm{MUSIC}}\right) \approx \frac{\delta_{1} \delta_{2}}{T}\left(\frac{1}{1-|\rho|^{2}}\right) \frac{\alpha_{N}}{(\Delta \theta)^{4}}\left(1+\sqrt{1+\frac{\delta_{3} T}{\delta_{1} \delta_{2}} \frac{(\Delta \theta)^{2}}{\beta_{N}}}\right),
$$

which also clearly increases with the magnitude of the correlation. For the NC MUSIC algorithm, the expression of mean of the threshold ASNRs (45)(46) which is not reachable will be illustrated in Section IV.

\section{ILLUSTRATIVE EXAMPLES}

This section illustrates the dependence of the threshold ASNR given by Results 1 and 2 on the non-Gaussianity of the noise and observations, and the phase and magnitude of the correlation of the complex circular and rectilinear sources by considering two illustration parts corresponding to the standard data and robust distribution models. Let us assume that two narrowband equal-power rectilinear correlated signal sources with power $\sigma_{x}^{2}$ impinge on a uniform linear array of $N=10$ sensors separated by a half-wavelength for which the steering vectors are $\mathbf{a}\left(\theta_{k}\right)$ is given by (43) where $\theta_{k}=\pi \sin \left(\omega_{k}\right)$, $k=1,2$, with $\omega_{k}$ is the DOAs relative to the normal of array broadside. The phases $\phi_{k}, k=1,2$ associated with different 
propagation delays are assumed fixed, but unknown during the array observation and the threshold ASNR depends only on $\Delta \phi \stackrel{\text { def }}{=} \phi_{1}-\phi_{2}$. The SNR is defined as $10 \log _{10}\left(\sigma_{x}^{2} / \sigma_{n}^{2}\right) \mathrm{dB}$.

\section{A. Standard data model}

In the first experiment, the noise $\mathbf{n}_{t}$ is either circular complex Student $t$-distributed with parameter $\nu>4$ to have finite fourth-order moment for which $\eta=\frac{\nu-2}{\nu-4}>1$, which has heavier tails than the Gaussian, or C-CG distributed (with $\eta=1$ obtained also for $\nu \rightarrow \infty$ ). We suppose the sources in model (5) consist of two multipaths issued from two independent BPSK modulated signals $e_{t, 1}$ and $e_{t, 2}$, for which we have $s_{t, 1}=e_{t, 1}$ and $s_{t, 2}=\rho^{\prime} e_{t, 1}+\sqrt{1-\rho^{\prime 2}} e_{t, 2}$ with $\rho^{\prime} \in(-1,+1)$. The two sources $x_{t, 1}$ and $x_{t, 2}$ are thus equal-powered with correlation $\rho=\rho^{\prime} e^{i \Delta \phi}$.

1) Influence of the non-Gaussianity of the noise: Figs. 1a and 1b exhibit the threshold ASNRs given by the Cox criterion and the Sharman and Durrani criterion as a function of the shape parameter $\nu$ for circular complex Student's $t$-distributed noise associated with the conventional MUSIC and NC MUSIC algorithms for uncorrelated sources. These figures show that the non-Gaussianity of the noise strongly impacts the threshold ASNR for large heavy-tailed distributions, i.e., when $\nu$ approaches 4 . We also note that the threshold ASNR loss is of the order of $2 \mathrm{~dB}$ when the C-CG distributed noise becomes circular complex Student's $t$-distributed for $\nu=4$.1. Furthermore, these figures confirm that the NC MUSIC algorithm greatly outperforms the conventional MUSIC algorithm for large phase separations.

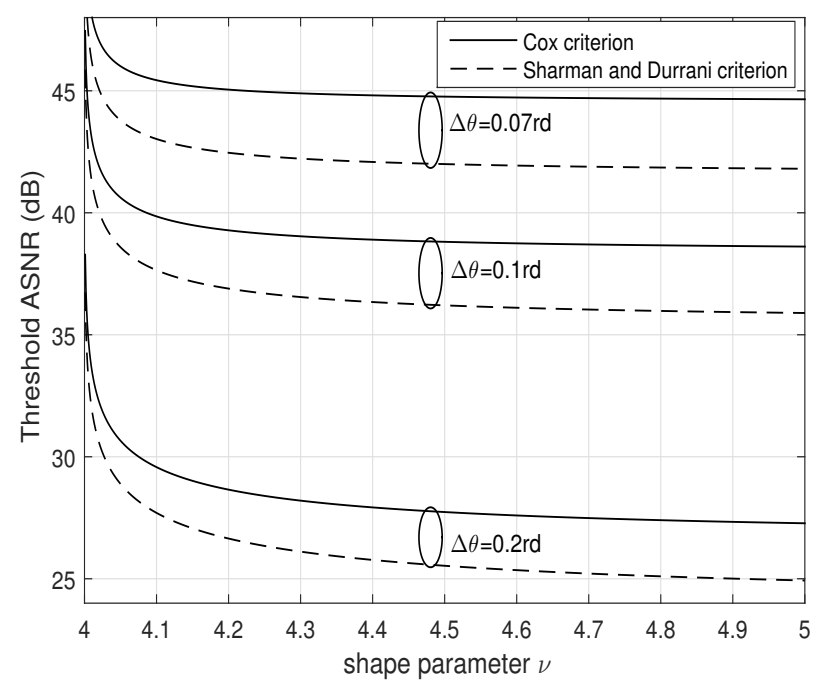

(a) Conventional MUSIC algorithm with $\rho=0$

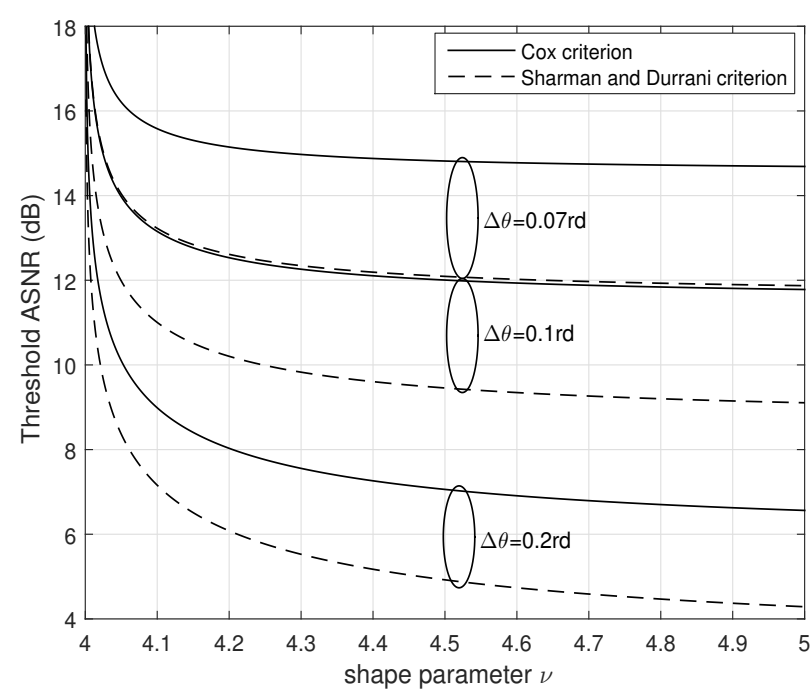

(b) NC-MUSIC algorithm with $\rho^{\prime}=0$ and $\Delta \phi=\pi / 2$

Fig. 1. Threshold ASNRs (42) (45) given by the Cox criterion and the Sharman and Durrani criterion as a function of the parameter $\nu$ associated with the conventional MUSIC and NC MUSIC algorithms for circular complex Student $t$-distributed noise.

\section{2) Influence of the correlation (magnitude and phase) for the conventional MUSIC algorithm with C-CG}

noise: Figs. $2 \mathrm{a}$ and $2 \mathrm{~b}$ show the crucial role played by the phase of the correlation. One can see that the resolution threshold ASNR of the conventional MUSIC algorithm is minimal [resp. maximal] for the phase of correlation $\angle \rho=\pi$ [resp., $\angle \rho=0$ ] as predicted by III-D2. Fig. 2a shows that there is a resolution gain of $3 \mathrm{~dB}$ when the sources are practically in phase opposition $(\angle \rho \approx \pi)$ compared to uncorrelated sources. We also naturally see a severe degradation for a large 
magnitude of the correlation ( $13 \mathrm{~dB}$ for $|\rho|=0.95$ ) for a zero phase with respect to uncorrelated sources. Fig. $2 \mathrm{~b}$ shows the threshold ASNR loss ratio $r_{1}=\left.\xi_{\mathrm{C}-\mathrm{MUSIC}}\right|_{|\rho|} /\left.\xi_{\mathrm{C}-\mathrm{MUSIC}}\right|_{|\rho|=0}$ varies with respect to $|\rho|$ in $\frac{1}{1-|\rho|}$ and $\frac{1}{1+|\rho|}$, for respectively $\angle \rho=0$ and $\angle \rho=\pi$ as depicted by III-D2.

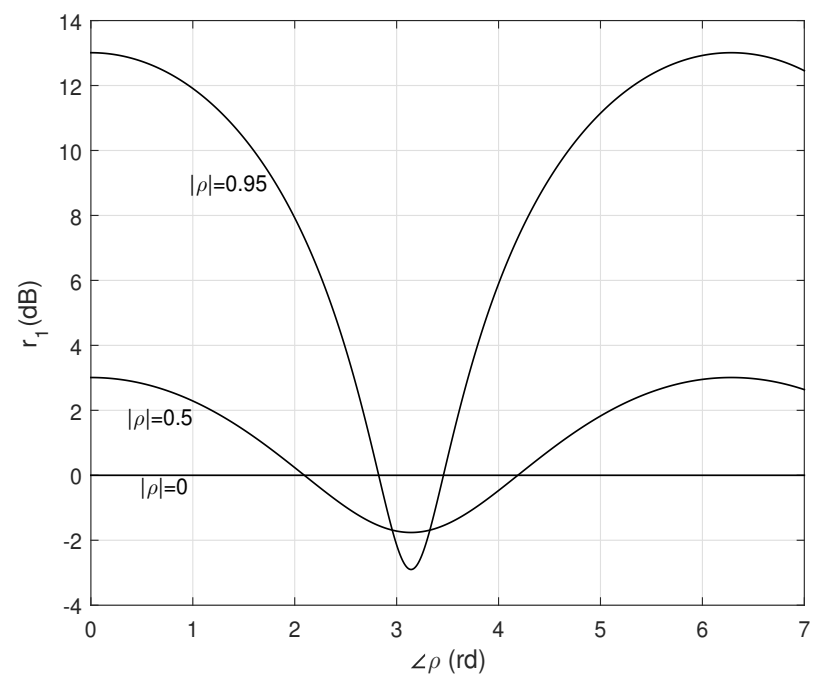

(a)

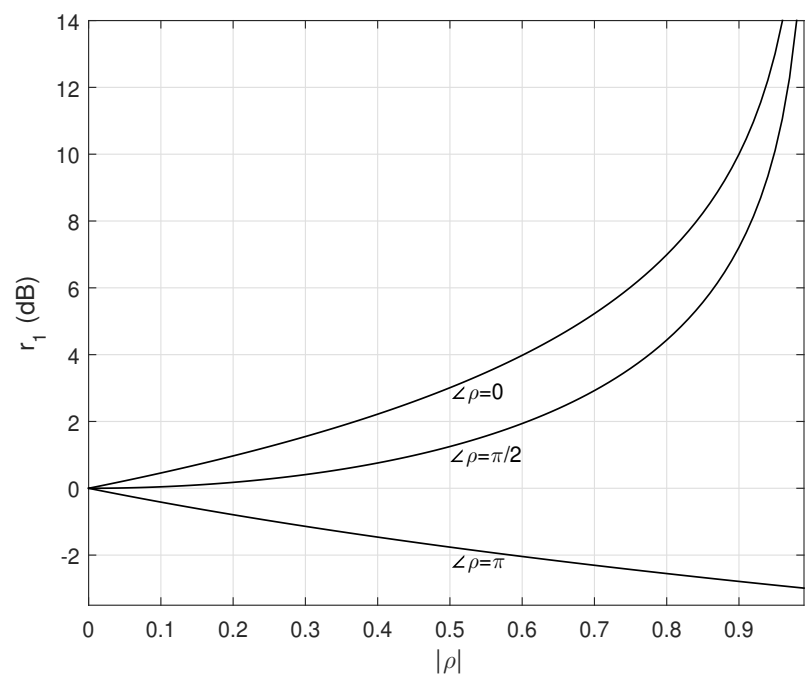

(b)

Fig. 2. Ratio $r_{1}=\left.\xi_{\mathrm{C}-\mathrm{MUSIC}}\right|_{|\rho|} /\left.\xi_{\mathrm{C}-\mathrm{MUSIC}}\right|_{|\rho|=0}$ given by the Cox criterion (42) for the conventional MUSIC algorithm with $\Delta \theta=0.05 \mathrm{rd}$ as a function of the angle (a) and magnitude (b) of the correlation.

\section{3) Influence of the correlation (magnitude and phase) for the NC MUSIC algorithm with C-CG noise:}

Fig. 3 illustrates that the threshold ASNR given for the NC MUSIC algorithm is maximum for $\Delta \phi=0$ rd mod $\pi$, but only minimum when $\Delta \phi=\pi / 2$ rd for uncorrelated sources. For correlated sources, the threshold ASNR is minimum for $\Delta \phi_{1}>\pi / 2$ for $\rho^{\prime}>0$ and for $\Delta \phi_{1}<\pi / 2$ for $\rho^{\prime}<0$ and this value of $\Delta \phi_{1}$ deviates from $\pi / 2$ when $\left|\rho^{\prime}\right|$ increases, as depicted by III-D3.

Fig. 4 shows that the threshold ASNR of the NC MUSIC algorithm with the particular value $\Delta \phi=\pi / 2$ does not depend on the sign of $\rho^{\prime}$ as depicted by III-D3 and naturally increases when $\Delta \theta$ decreases.

4) Mean of the threshold ASNRs with respect to the correlation phase: Figs. 5a and 5b represent the mean of the threshold ASNR with respect to the correlation phase given by the Cox criterion for the conventional MUSIC and NC MUSIC algorithms, respectively, with C-CG distributed noise, as a function of the magnitude of the correlation. This mean is derived from (48) for the conventional MUSIC algorithm and by averaging 12000 realizations for both conventional MUSIC and NC MUSIC algorithms. Fig. 5a shows that the approximate value of the threshold ASNR given by the closed-form expressions (48) is very close to the mean derived from (39) and (40). We clearly see a great degradation in resolution for correlated sources compared to uncorrelated sources (about $7 \mathrm{~dB}$ for $|\rho|=0.9$ for both algorithms). This figure also shows the advantage of using the NC MUSIC algorithm rather than the conventional MUSIC algorithm for rectilinear sources. 


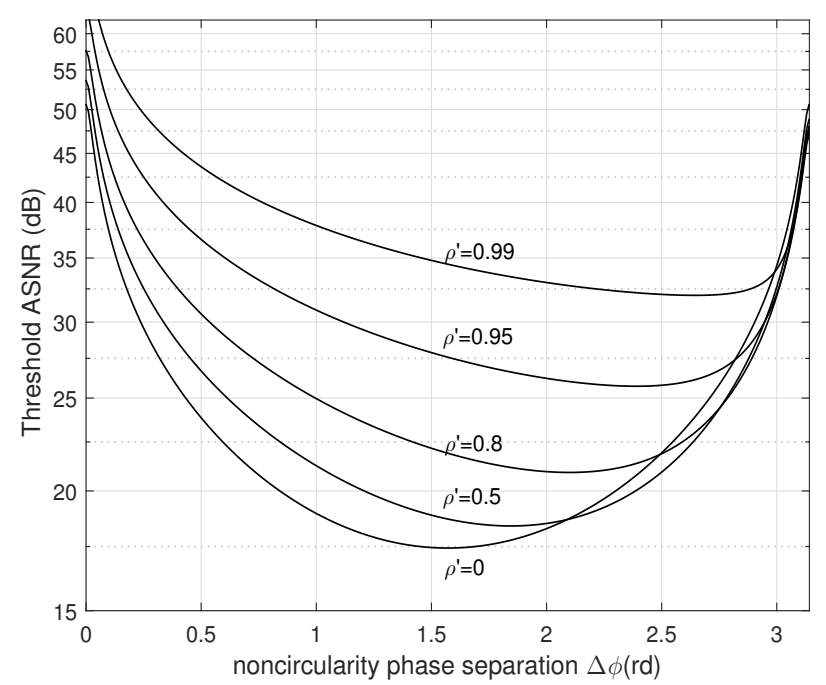

(a)

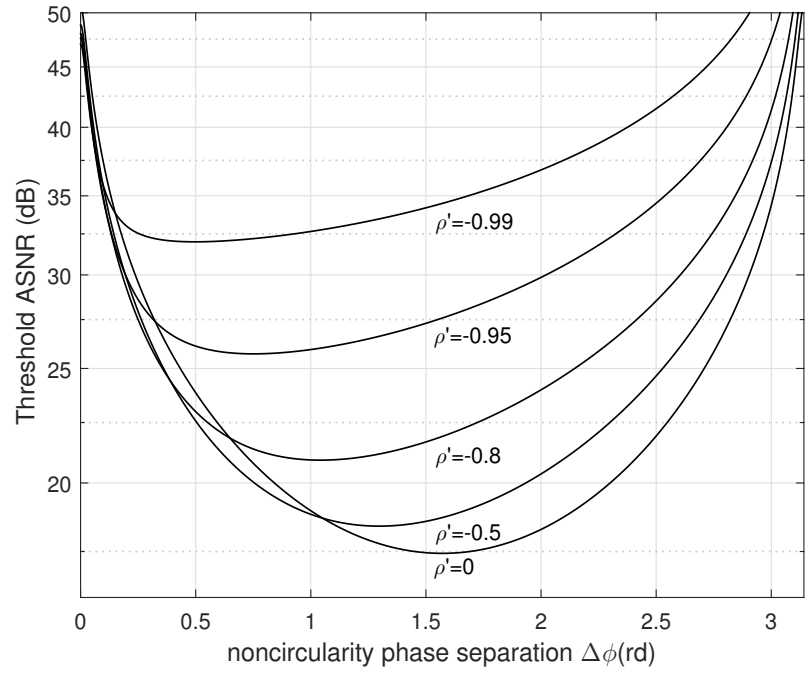

(b)

Fig. 3. Threshold ASNR given by the Cox criterion (45) for the NC MUSIC algorithm with C-CG distributed noise as a function of the noncircularity phase separation $\Delta \phi$ for $\Delta \theta=0.05 \mathrm{rd}$ for negative (a) and positive (b) values of $\rho^{\prime}$ as shown in (8).

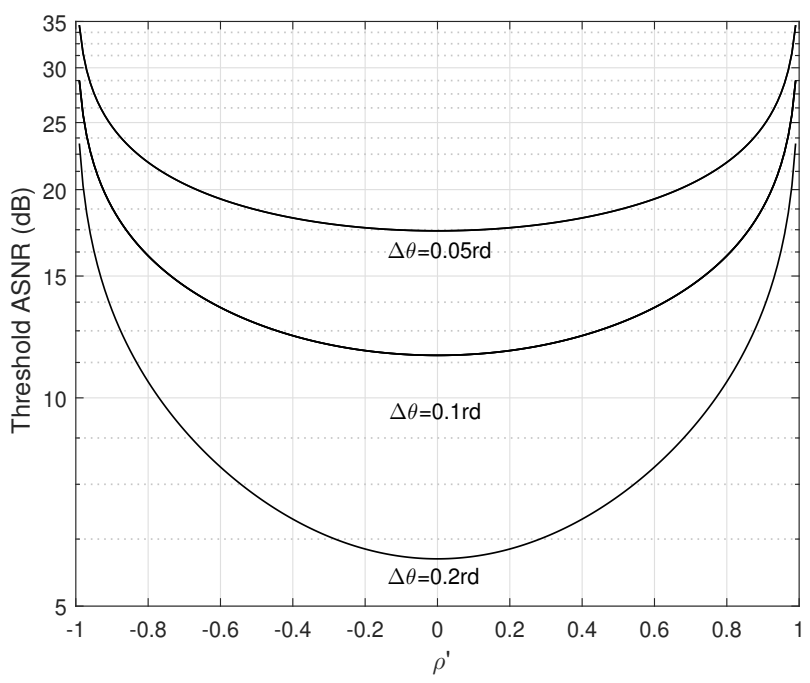

Fig. 4. Threshold ASNR given by the Cox criterion (45) for the NC MUSIC algorithm with C-CG distributed noise as a function of $\rho^{\prime}$ for $\Delta \phi=\pi / 2$.

\section{B. Robust distribution model}

In this second experiment, we assume that the observations $\mathbf{y}_{t}$ follow either a circular or a NC complex Student $t$ distribution with parameter $\nu>4$, with associated structured covariance $\mathbf{R}_{y}$ given by (3), or extended covariance $\mathbf{R}_{\tilde{y}}$ given by (7), respectively. These robust distribution models can be considered as second-order approximative models of the actual ones.

We consider here three estimates of covariance and extended covariance based on the circular or NC complex Student $t$-distribution: the complex Student's ML, $M$-estimator and the complex Tyler's $M$-estimator for which the associated 


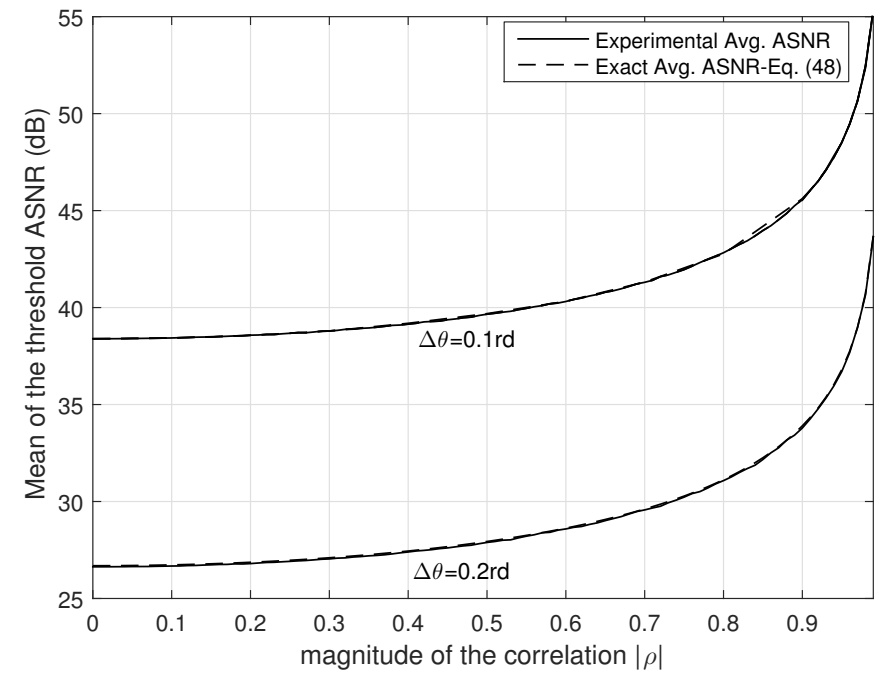

(a)

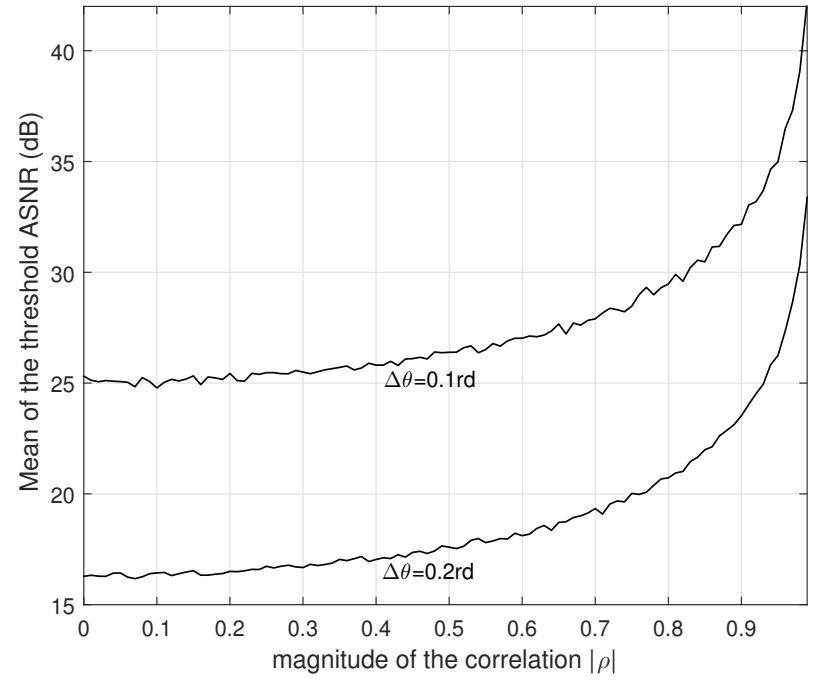

(b)

Fig. 5. Mean of the threshold ASNR with respect to the correlation phase given by the Cox criterion (42),(45) for the conventional MUSIC algorithm (a) and NC MUSIC algorithm (b) with C-CG distributed noise, as a function of the magnitude of the correlation.

weight functions $\psi(t)$ and $u(t)$ are respectively defined in [28] by $\psi(t)=\frac{2 N+\nu}{\nu+2 t}$ and $u(t)=\frac{N}{t}$. The SCM estimator corresponding to the ML in the Gaussian case is obtained with $u(t)=1$. The parameters $\vartheta_{1}$ and $\sigma_{u}$ of this complex Student $t$-distribution are also given by $\vartheta_{1}=\frac{N+\nu / 2+1}{N+\nu / 2}$ and $\sigma_{u}=1$ for ML $M$-estimator, $\vartheta_{1}=\frac{N+1}{N}$ and $\sigma_{u}=1$ for Tyler's $M$-estimate and by $\vartheta_{1}=\eta=\frac{\nu-2}{\nu-4}$ and $\sigma_{u}=1$ for the SCM [30].

Fig. 6 exhibits the threshold ASNRs given by the Cox criterion for circular [resp., NC] complex Student $t$-distributed observations associated with the conventional [resp., NC] MUSIC algorithms, based on ML $M$-estimate, Tyler's $M$ estimate and SCM of the covariance [resp., extended covariance], as a function of $\Delta \theta$. We see that for heavy-tailed distributions $(\nu=4.1)$, the threshold ASNRs provided by Tyler's $M$-estimate reaches that of the ML $M$-estimate and outperforms those of the SCM by about 10dB. Furthermore, naturally, the NC MUSIC algorithm applied to rectilinear sources outperforms the conventional MUSIC algorithm applied to circular sources, associated with the same structured covariance $\mathbf{R}_{y}$ (3). Fig. 7 shows estimation of probability of resolution related to the Cox and to the Sharman and Durrani criteria for NC complex Student $t$-distributed observation models (with $\nu=4.1$ ) obtained by Monte Carlo simulations ${ }^{6}$ for highly correlated sources $\left(\rho^{\prime}=0.95\right)$ and $\Delta \theta=0.2 \mathrm{rd}$. 1000 independent Monte Carlo runs have been performed where the number of snapshots is fixed at $T=500$. We see that the ASNR threshold given by our non-probabilistic approach based on the mean null spectrum $g_{T}(\theta)$ confirms the results of Fig. 6 .

\footnotetext{
${ }^{6}$ In each simulation trial, the two sources are considered resolved for the Sharman and Durrani criterion if $g_{T}^{\operatorname{Alg}_{N C}}\left(\theta_{m}+0.002 \Delta \theta\right)+$ $g_{T}^{\operatorname{Alg}_{N C}}\left(\theta_{m}-0.002 \Delta \theta\right)-2 g_{T}^{\operatorname{Alg}_{N C}}\left(\theta_{m}\right)<0$.
} 


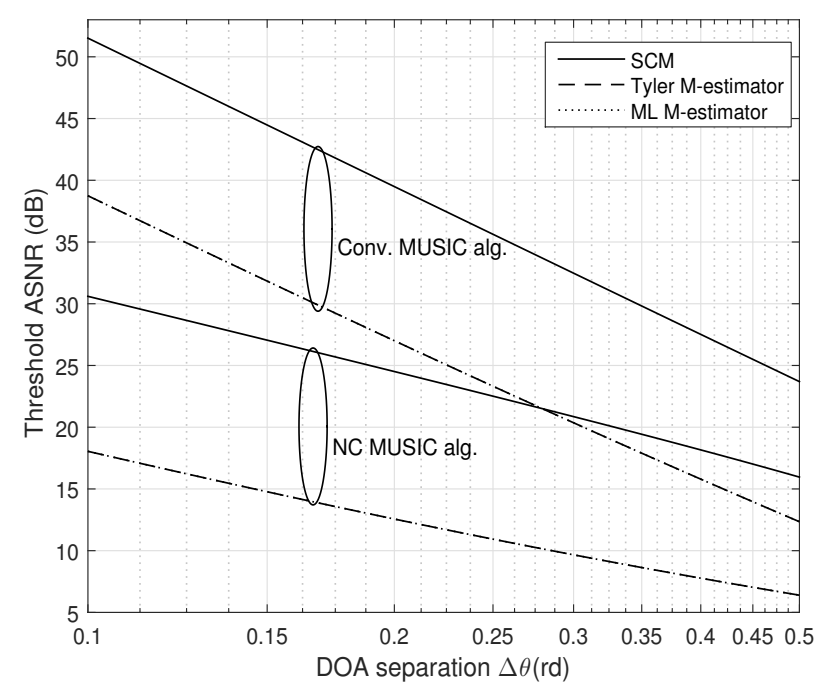

(a) $|\rho|=\rho^{\prime}=0$

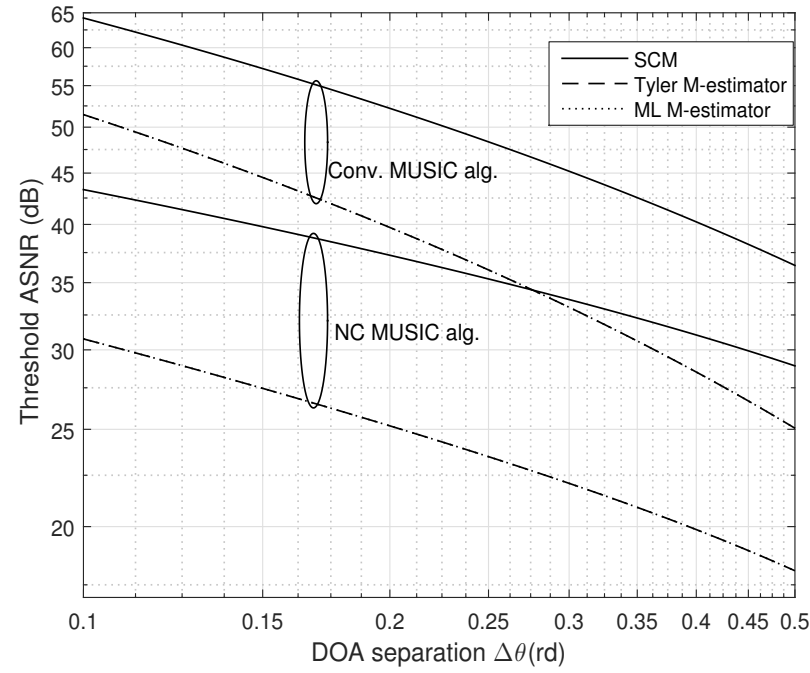

(b) $|\rho|=\rho^{\prime}=0.95$

Fig. 6. Threshold ASNRs (42),(45) given by the Cox criterion for circular [resp., NC] complex Student $t$-distributed observations with $\nu=4.1$ associated with the conventional [resp., NC] MUSIC algorithms, based on ML $M$-estimate, Tyler's $M$-estimate and SCM of the covariance [resp., extended sample covariance], as function of $\Delta \theta$, with $\Delta \phi=\pi / 6$.

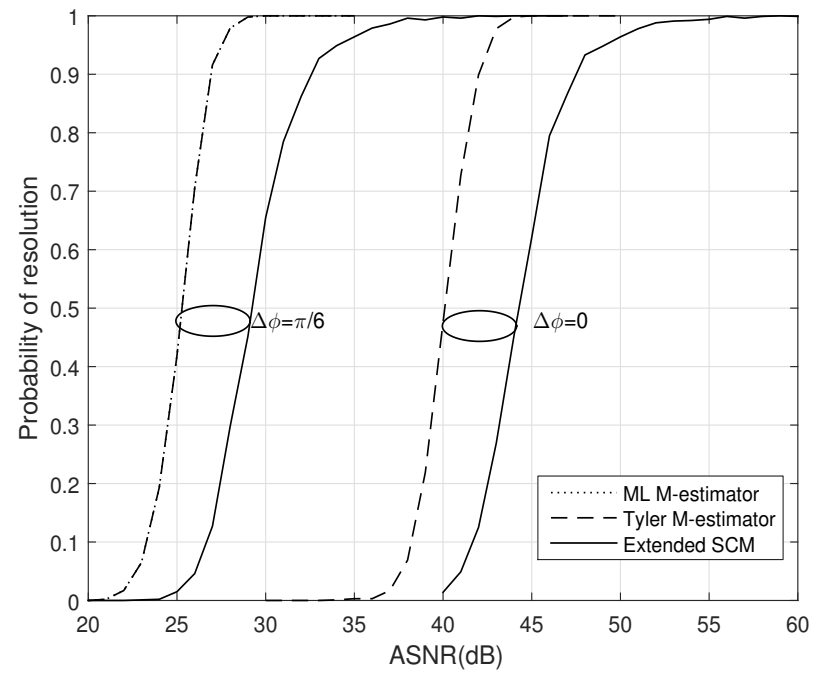

(a) Cox criterion

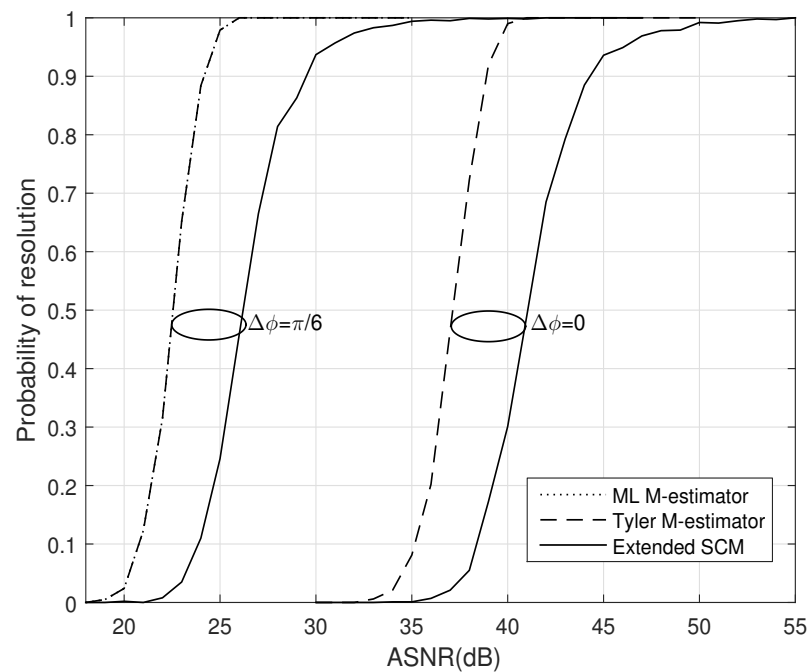

(b) Sharman and Durrani criterion

Fig. 7. Probability of resolution as function of the ASNR for NC complex Student' $t$-distributed observation models with $\nu=4.1$, $\rho^{\prime}=0.95$ and $\Delta \theta=0.2 \mathrm{rd}$; given by the Cox and the Sharman and Durrani criteria associated with the NC MUSIC algorithm for two values of $\Delta \phi$.

\section{CONCLUSION}

In this paper, we have derived interpretable unified closed-form expressions for the threshold ASNR along the Cox and the Sharman and Durrani criteria associated with the conventional MUSIC and NC MUSIC algorithms in the context of arbitrary circular or rectilinear distributed correlated sources and circular CES distributed noise, as well as of C-CES and NC-CES distributed observations. Using these expressions, we investigated the impact of the non-Gaussianity of the noise 
and observations, as well as of the phase and magnitude of the correlation of the sources. In particular, we proved for the first time that the phase of the correlation, which is the non-circularity phase separation for rectilinear sources, may have a strong impact on the resolution and a zero phase may lead to overly optimistic resolution. Otherwise, we quantified the resolution benefit provided when the SCM is replaced by $M$-estimates of the covariance matrix for CES observations.

\section{APPENDIX}

\section{A. Proof of Lemma 1}

We will make use of the following relations proved in [48, chp.16] which hold for any conformable matrices A, B, C, and $\mathbf{D}$ and vectors $\mathbf{a}$ and $\mathbf{b}$.

$$
\begin{gathered}
\operatorname{vec}(\mathbf{A B C})=\left(\mathbf{C}^{T} \otimes \mathbf{A}\right) \operatorname{vec}(\mathbf{B}), \\
(\mathbf{A} \otimes \mathbf{B})(\mathbf{C} \otimes \mathbf{D})=\mathbf{A} \mathbf{C} \otimes \mathbf{B D}, \\
\operatorname{vec}\left(\mathbf{a b}^{T}\right)=\mathbf{b} \otimes \mathbf{a}, \\
\operatorname{Tr}(\mathbf{A B})=\operatorname{vec}^{T}\left(\mathbf{A}^{T}\right) \operatorname{vec}(\mathbf{B}), \\
\mathbf{K}(\mathbf{A} \otimes \mathbf{B})=(\mathbf{B} \otimes \mathbf{A}) \mathbf{K}, \\
\operatorname{vec}(\mathbf{A} \otimes \mathbf{B})=(\mathbf{I} \otimes \mathbf{K} \otimes \mathbf{I})[\operatorname{vec}(\mathbf{A}) \otimes \operatorname{vec}(\mathbf{B})], \\
(\mathbf{I} \otimes \mathbf{K} \otimes \mathbf{I}) \operatorname{vec}(\mathbf{K})=\operatorname{vec}(\mathbf{K}) .
\end{gathered}
$$

Let us first consider the standard data model and start to prove (25). Using the vectorization operator, it follows form (49) that

$$
\operatorname{vec}\left[\mathrm{E}\left(\delta \mathbf{R}_{y, T} \mathbf{B}_{1} \delta \mathbf{R}_{y, T}\right)\right]=\mathrm{E}\left(\delta \mathbf{R}_{y, T}^{T} \otimes \delta \mathbf{R}_{y, T}\right) \operatorname{vec}\left(\mathbf{B}_{1}\right),
$$

with $\delta \mathbf{R}_{y, T} \stackrel{\text { def }}{=} \mathbf{R}_{y, T}-\mathbf{R}_{y}$ gives after straightforward manipulations

$$
\begin{aligned}
\mathrm{E}\left(\delta \mathbf{R}_{y, T}^{T} \otimes \delta \mathbf{R}_{y, T}\right) & =\frac{1}{T}\left(\mathrm{E}\left(\mathbf{y}_{t}^{*} \mathbf{y}_{t}^{T} \otimes \mathbf{y}_{t} \mathbf{y}_{t}^{H}\right)-\mathbf{R}_{y}^{T} \otimes \mathbf{R}_{y}\right) \\
& =\frac{1}{T}\left(\mathrm{E}\left[\operatorname{vec}\left(\mathbf{y}_{t} \mathbf{y}_{t}^{H}\right) \operatorname{vec}^{H}\left(\mathbf{y}_{t} \mathbf{y}_{t}^{H}\right)\right]-\mathbf{R}_{y}^{T} \otimes \mathbf{R}_{y}\right) \\
& =\frac{1}{T}\left(T \mathbf{R}_{r_{y}}+\operatorname{vec}\left(\mathbf{R}_{y}\right) \operatorname{vec}^{H}\left(\mathbf{R}_{y}\right)-\mathbf{R}_{y}^{T} \otimes \mathbf{R}_{y}\right),
\end{aligned}
$$

where (50) and (51) is used in the second equality and where $\mathbf{R}_{r_{y}}$ is also the covariance of the random vector $\frac{1}{\sqrt{T}} \operatorname{vec}\left(\mathbf{y}_{t} \mathbf{y}_{t}^{H}\right)$. Using its expression in (16) and(18), we get

$$
\begin{aligned}
\mathrm{E}\left(\delta \mathbf{R}_{y, T}^{T} \otimes \delta \mathbf{R}_{y, T}\right) & =\frac{1}{T}\left(\operatorname{vec}\left(\mathbf{R}_{y}\right) \operatorname{vec}^{H}\left(\mathbf{R}_{y}\right)+\mathbf{K}\left(\mathbf{C}_{y} \otimes \mathbf{C}_{y}^{*}\right)+\left(\mathbf{A}^{*} \otimes \mathbf{A}\right) \mathbf{Q}_{x}\left(\mathbf{A}^{T} \otimes \mathbf{A}^{H}\right)\right. \\
& \left.+\sigma_{n}^{4}(\eta-1)\left[\mathbf{I}+\operatorname{vec}(\mathbf{I}) \operatorname{vec}^{T}(\mathbf{I})\right]\right) .
\end{aligned}
$$


Plugging (58) into (56) and using (49), (52) and (53), (25) is proved. The proof of (26) follows the same steps as the proof of (25) using (17) and (19).

Consider now the robust distribution model and let us start to prove (27) for the conventional MUSIC algorithm. From the asymptotic distribution of $\mathbf{R}_{y, T}^{u} \stackrel{\text { def }}{=} \sigma_{u}^{-1} \boldsymbol{\Gamma}_{y, T}^{u}$, we deduce that

$$
\mathrm{E}\left[\operatorname{vec}\left(\delta \mathbf{R}_{y, T}^{u}\right) \operatorname{vec}^{H}\left(\delta \mathbf{R}_{y, T}^{u}\right)\right] \approx \frac{1}{T \sigma_{u}^{2}} \mathbf{R}_{\Gamma_{y}^{u}},
$$

for large enough $T$, where $\mathbf{R}_{\Gamma_{y}^{u}}$ is given by (20). By vectorization of (59) and using (51), we get

$$
\mathrm{E}\left[\operatorname{vec}\left(\delta \mathbf{R}_{y, T}^{u}{ }^{T}\right) \otimes \operatorname{vec}\left(\delta \mathbf{R}_{y, T}^{u}\right)\right] \approx \frac{1}{T \sigma_{u}^{2}}\left[\vartheta_{1} \operatorname{vec}\left(\mathbf{R}_{y}^{*} \otimes \mathbf{R}_{y}\right)+\vartheta_{2} \operatorname{vec}\left(\mathbf{R}_{y}^{*}\right) \otimes \operatorname{vec}\left(\mathbf{R}_{y}\right)\right] .
$$

Using (54), we deduce that (60) is equivalent to

$$
\mathrm{E}\left(\delta \mathbf{R}_{y, T}^{u}{ }^{T} \otimes \delta \mathbf{R}_{y, T}^{u}\right) \approx \frac{1}{T \sigma_{u}^{2}}\left[\vartheta_{1} \operatorname{vec}\left(\mathbf{R}_{y}\right) \operatorname{vec}^{H}\left(\mathbf{R}_{y}\right)+\vartheta_{2}\left(\mathbf{R}_{y}^{*} \otimes \mathbf{R}_{y}\right)\right] .
$$

Plugging (61) into (56) where $\delta \mathbf{R}_{y, T}$ is replaced by $\delta \mathbf{R}_{y, T}^{u}$ and using (49) and (52), (27) is proved.

The proof of (28) for the NC MUSIC algorithm begins in the same way, where (60) yields

$$
\operatorname{E}\left[\operatorname{vec}\left(\delta \mathbf{R}_{\tilde{y}, T}^{u}{ }^{T}\right) \otimes \operatorname{vec}\left(\delta \mathbf{R}_{\tilde{y}, T}^{u}\right)\right] \approx \frac{1}{T \sigma_{u}^{2}}\left[\vartheta_{1}\left\{\operatorname{vec}\left(\mathbf{R}_{\tilde{y}}^{*} \otimes \mathbf{R}_{\tilde{y}}\right)+\operatorname{vec}\left(\mathbf{K}\left(\mathbf{C}_{\tilde{y}} \otimes \mathbf{C}_{\tilde{y}}^{*}\right)\right)\right\}+\vartheta_{2} \operatorname{vec}\left(\mathbf{R}_{\tilde{y}}^{*}\right) \otimes \operatorname{vec}\left(\mathbf{R}_{\tilde{y}}\right)\right] .
$$

But now the rest of the proof is more complex because the permutation matrix $\mathbf{K}$ comes into (62). To exclude it, we premultiply to the left (62) by $\mathbf{I} \otimes \mathbf{K} \otimes \mathbf{I}$, and thanks to (54), we obtain

$$
\operatorname{vec}\left[\mathrm{E}\left(\delta \mathbf{R}_{\tilde{y}, T}^{u} T^{T} \otimes \delta \mathbf{R}_{\tilde{y}, T}^{u}\right)\right] \approx \frac{1}{T \sigma_{u}^{2}}\left[\vartheta_{1}\left\{\operatorname{vec}\left(\mathbf{R}_{\tilde{y}}^{*}\right) \otimes \operatorname{vec}\left(\mathbf{R}_{\tilde{y}}\right)+(\mathbf{I} \otimes \mathbf{K} \otimes \mathbf{I}) \operatorname{vec}\left(\mathbf{K}\left(\mathbf{C}_{\tilde{y}} \otimes \mathbf{C}_{\tilde{y}}^{*}\right)\right)\right\}+\vartheta_{2} \operatorname{vec}\left(\mathbf{R}_{\tilde{y}}^{*} \otimes \mathbf{R}_{\tilde{y}}\right)\right] .
$$

We then prove after some algebraic manipulations, using (49) and (55), that $(\mathbf{I} \otimes \mathbf{K} \otimes \mathbf{I}) \operatorname{vec}\left(\mathbf{K}\left(\mathbf{C}_{\tilde{y}} \otimes \mathbf{C}_{\tilde{y}}^{*}\right)\right)=\operatorname{vec}\left(\mathbf{K}\left(\mathbf{C}_{\tilde{y}} \otimes\right.\right.$ $\left.\mathbf{C}_{\tilde{y}}^{*}\right)$ ). It follows from (51) that $\operatorname{vec}\left(\mathbf{R}_{\tilde{y}}^{*}\right) \otimes \operatorname{vec}\left(\mathbf{R}_{\tilde{y}}\right)=\operatorname{vec}\left[\operatorname{vec}\left(\mathbf{R}_{\tilde{y}}\right) \operatorname{vec}^{T}\left(\mathbf{R}_{\tilde{y}}^{T}\right)\right]$, and hence (63) is equivalent to

$$
\mathrm{E}\left(\delta \mathbf{R}_{\tilde{y}, T}^{u}{ }^{T} \otimes \delta \mathbf{R}_{\tilde{y}, T}^{u}\right) \approx \frac{1}{T \sigma_{u}^{2}}\left[\vartheta_{1}\left\{\operatorname{vec}\left(\mathbf{R}_{\tilde{y}}\right) \operatorname{vec}^{T}\left(\mathbf{R}_{\tilde{y}}^{T}\right)+\mathbf{K}\left(\mathbf{C}_{\tilde{y}} \otimes \mathbf{C}_{\tilde{y}}^{*}\right)\right\}+\vartheta_{2}\left(\mathbf{R}_{\tilde{y}}^{*} \otimes \mathbf{R}_{\tilde{y}}\right)\right]
$$

Plugging (64) into (56) and using (49), (52) and (53), (28) is proved.

\section{B. Proof of relations (37) and (38)}

To simplify the derivation of $\mathrm{E}\left(g_{T}^{\operatorname{Alg}_{N C}}(\theta)\right)$, let us introduce $\mathbf{M} \stackrel{\text { def }}{=} \mathbf{a}(\theta) \mathbf{a}^{H}(\theta)$ and $\mathbf{N} \stackrel{\text { def }}{=} \mathbf{a}(\theta) \mathbf{a}^{T}(\theta)$ for which we have:

$$
g_{T}^{\mathrm{Alg}}{ }_{\mathrm{NC}}(\theta)=\operatorname{Tr}\left(\boldsymbol{\Pi}_{1, T} \mathbf{M}\right) \operatorname{Tr}\left(\mathbf{\Pi}_{1, T} \mathbf{M}\right)-\operatorname{Tr}\left(\boldsymbol{\Pi}_{2, T}^{*} \mathbf{N}\right) \operatorname{Tr}\left(\boldsymbol{\Pi}_{2, T} \mathbf{N}^{*}\right),
$$

which gives with $\delta \boldsymbol{\Pi}_{1, T} \stackrel{\text { def }}{=} \boldsymbol{\Pi}_{1, T}-\boldsymbol{\Pi}_{1}$ and $\delta \boldsymbol{\Pi}_{2, T} \stackrel{\text { def }}{=} \boldsymbol{\Pi}_{2, T}-\boldsymbol{\Pi}_{2}$

$$
\begin{aligned}
\mathrm{E}\left(g_{T}^{\operatorname{Alg}{ }_{\mathbf{N C}}}(\theta)\right) & =g^{\operatorname{Alg}_{\mathrm{NC}}}(\theta) \\
& +2 \operatorname{Tr}\left[\mathrm{E}\left(\delta \boldsymbol{\Pi}_{1, T}\right) \mathbf{M}\right] \operatorname{Tr}\left(\boldsymbol{\Pi}_{1} \mathbf{M}\right)-2 \operatorname{Re}\left(\operatorname{Tr}\left[\mathrm{E}\left(\delta \boldsymbol{\Pi}_{2, T}\right) \mathbf{N}^{*}\right] \operatorname{Tr}\left(\boldsymbol{\Pi}_{2}^{*} \mathbf{N}\right)\right) \\
& +\mathrm{E}\left[\operatorname{Tr}\left(\delta \boldsymbol{\Pi}_{1, T} \mathbf{M}\right) \operatorname{Tr}\left(\delta \boldsymbol{\Pi}_{1, T} \mathbf{M}\right)\right]-\mathrm{E}\left[\operatorname{Tr}\left(\delta \boldsymbol{\Pi}_{2, T}^{*} \mathbf{N}\right) \operatorname{Tr}\left(\delta \boldsymbol{\Pi}_{2, T} \mathbf{N}^{*}\right)\right]
\end{aligned}
$$


The first-order terms of (65) are deduced from (31) and (32), which gives from $\operatorname{Tr}[\widetilde{\mathbf{\Pi}})=2 \operatorname{Tr}\left[\boldsymbol{\Pi}_{1}\right)=2 N-2$ where $\mathbf{a} \stackrel{\text { def }}{=} \mathbf{a}(\theta)$ (for sake of brevity)

$$
\begin{aligned}
\operatorname{Tr}\left[\mathrm{E}\left(\delta \boldsymbol{\Pi}_{1, T}\right) \mathbf{M}\right] & \approx \frac{1}{T}\left((2 N-2)\left(\mathbf{a}^{H} \mathbf{U}_{1} \mathbf{a}\right)-2 \operatorname{Tr}\left(\mathbf{U}_{1}\right)\left(\mathbf{a}^{H} \boldsymbol{\Pi}_{1} \mathbf{a}\right)\right. \\
& \left.+(\eta-1)\left[(2 N-2)\left(\mathbf{a}^{H} \mathbf{U}_{1}^{\prime} \mathbf{a}\right)-2 \operatorname{Tr}\left(\mathbf{U}_{1}^{\prime}\right)\left(\mathbf{a}^{H} \boldsymbol{\Pi}_{1} \mathbf{a}\right)\right]\right), \\
\operatorname{Tr}\left[\mathrm{E}\left(\delta \boldsymbol{\Pi}_{2, T}\right) \mathbf{N}^{*}\right] & \approx \frac{1}{T}\left((2 N-2)\left(\mathbf{a}^{H} \mathbf{U}_{2} \mathbf{a}^{*}\right)-2 \operatorname{Tr}\left(\mathbf{U}_{1}\right) \mathbf{a}^{H} \boldsymbol{\Pi}_{2} \mathbf{a}^{*}\right. \\
& \left.+(\eta-1)\left[(2 N-2)\left(\mathbf{a}^{H} \mathbf{U}_{2}^{\prime} \mathbf{a}^{*}\right)-2 \operatorname{Tr}\left(\mathbf{U}_{1}^{\prime}\right)\left(\mathbf{a}^{H} \boldsymbol{\Pi}_{2} \mathbf{a}^{*}\right)\right]\right) .
\end{aligned}
$$

Introducing these expressions into (65), the first-order terms are given by

$$
\begin{aligned}
2 \operatorname{Tr}\left[\mathrm{E}\left(\delta \boldsymbol{\Pi}_{1, T}\right) \mathbf{M}\right] \operatorname{Tr}\left(\boldsymbol{\Pi}_{1} \mathbf{M}\right) & \approx \frac{2}{T}\left((2 N-2)\left(\mathbf{a}^{H} \mathbf{U}_{1} \mathbf{a}\right)\left(\mathbf{a}^{H} \boldsymbol{\Pi}_{1} \mathbf{a}\right)-2 \operatorname{Tr}\left(\mathbf{U}_{1}\right)\left(\mathbf{a}^{H} \boldsymbol{\Pi}_{1} \mathbf{a}\right)^{2}\right. \\
& \left.+(\eta-1)\left[(2 N-2)\left(\mathbf{a}^{H} \mathbf{U}_{1}^{\prime} \mathbf{a}\right)\left(\mathbf{a}^{H} \boldsymbol{\Pi}_{1} \mathbf{a}\right)-2 \operatorname{Tr}\left(\mathbf{U}_{1}^{\prime}\right)\left(\mathbf{a}^{H} \boldsymbol{\Pi}_{1} \mathbf{a}\right)^{2}\right]\right), \\
2 \operatorname{Re}\left(\operatorname{Tr}\left[\mathrm{E}\left(\delta \boldsymbol{\Pi}_{2, T}\right) \mathbf{N}^{*}\right] \operatorname{Tr}\left(\boldsymbol{\Pi}_{2}^{*} \mathbf{N}\right)\right. & \approx \frac{2}{T}\left((2 N-2) \operatorname{Re}\left(\left(\mathbf{a}^{H} \mathbf{U}_{2} \mathbf{a}^{*}\right)\left(\mathbf{a}^{T} \boldsymbol{\Pi}_{2}^{*} \mathbf{a}\right)\right)-2 \operatorname{Tr}\left(\mathbf{U}_{1}\right)\left|\mathbf{a}^{H} \boldsymbol{\Pi}_{2} \mathbf{a}^{*}\right|^{2}\right), \\
& \left.+(\eta-1)\left[(2 N-2) \operatorname{Re}\left(\left(\mathbf{a}^{H} \mathbf{U}_{2}^{\prime} \mathbf{a}^{*}\right)\left(\mathbf{a}^{T} \boldsymbol{\Pi}_{2}^{*} \mathbf{a}\right)\right)-2 \operatorname{Tr}\left(\mathbf{U}_{1}^{\prime}\right)\left|\mathbf{a}^{H} \boldsymbol{\Pi}_{2} \mathbf{a}^{*}\right|^{2}\right]\right) .
\end{aligned}
$$

The second-order terms of (65) are given by

$$
\begin{aligned}
\mathrm{E}\left[\operatorname{Tr}\left(\delta \boldsymbol{\Pi}_{1, T} \mathbf{M}\right) \operatorname{Tr}\left(\delta \boldsymbol{\Pi}_{1, T} \mathbf{M}\right)\right] & =\operatorname{vec}^{T}\left(\mathbf{M}^{T}\right) \mathrm{E}\left(\operatorname{vec}\left(\delta \boldsymbol{\Pi}_{1, T}\right) \operatorname{vec}^{H}\left(\delta \boldsymbol{\Pi}_{1, T}\right)\right) \operatorname{vec}(\mathbf{M}) \\
& \approx \frac{1}{T} \operatorname{vec}^{T}\left(\mathbf{M}^{T}\right) \mathbf{C}_{\Pi_{1}} \operatorname{vec}(\mathbf{M}), \\
\mathrm{E}\left[\operatorname{Tr}\left(\delta \boldsymbol{\Pi}_{2, T}^{*} \mathbf{N}\right) \operatorname{Tr}\left(\delta \boldsymbol{\Pi}_{2, T} \mathbf{N}^{*}\right)\right] & =\operatorname{vec}^{T}(\mathbf{N}) \mathrm{E}\left(\operatorname{vec}\left(\delta \boldsymbol{\Pi}_{2, T}^{*}\right) \operatorname{vec}^{T}\left(\delta \boldsymbol{\Pi}_{2, T}\right)\right) \operatorname{vec}\left(\mathbf{N}^{*}\right) \\
& \approx \frac{1}{T} \operatorname{vec}^{T}(\mathbf{N}) \mathbf{C}_{\Pi_{2}}^{*} \operatorname{vec}\left(\mathbf{N}^{*}\right),
\end{aligned}
$$

where $\mathbf{C}_{\Pi_{1}}$ and $\mathbf{C}_{\Pi_{2}}$ denote the covariance matrices of the asymptotic distribution of $\boldsymbol{\Pi}_{1, T}$ and $\boldsymbol{\Pi}_{2, T}$, respectively, deduced from the covariance matrices $\mathbf{C}_{\widetilde{\Pi}}$ of the asymptotic distribution of $\widetilde{\boldsymbol{\Pi}}_{T}$ given from [47] by:

$$
\mathbf{C}_{\widetilde{\Pi}}=(\mathbf{I}+\mathbf{K}(\mathbf{J} \otimes \mathbf{J}))\left(\left(\widetilde{\boldsymbol{\Pi}}^{*} \otimes \widetilde{\mathbf{U}}\right)+\left(\widetilde{\mathbf{U}}^{*} \otimes \widetilde{\mathbf{\Pi}}\right)\right)+(\eta-1)(\mathbf{I}+\mathbf{K}(\mathbf{J} \otimes \mathbf{J}))\left(\left(\widetilde{\boldsymbol{\Pi}}^{*} \otimes \widetilde{\mathbf{U}}^{\prime}\right)+\left(\widetilde{\mathbf{U}}^{*} \otimes \widetilde{\mathbf{\Pi}}\right)\right),
$$

that implies:

$$
\begin{aligned}
\mathbf{C}_{\Pi_{1}} & =\left(\boldsymbol{\Pi}_{1}^{*} \otimes \mathbf{U}_{1}\right)+\left(\mathbf{U}_{1}^{*} \otimes \boldsymbol{\Pi}_{1}\right)+\mathbf{K}\left[\left(\boldsymbol{\Pi}_{2} \otimes \mathbf{U}_{2}^{*}\right)+\left(\mathbf{U}_{2} \otimes \boldsymbol{\Pi}_{2}^{*}\right)\right] \\
& +(\eta-1)\left(\left(\boldsymbol{\Pi}_{1}^{*} \otimes \mathbf{U}_{1}^{\prime}\right)+\left(\mathbf{U}_{1}^{\prime *} \otimes \boldsymbol{\Pi}_{1}\right)+\mathbf{K}\left[\left(\boldsymbol{\Pi}_{2} \otimes \mathbf{U}_{2}^{\prime *}\right)+\left(\mathbf{U}_{2}^{\prime} \otimes \boldsymbol{\Pi}_{2}^{*}\right)\right]\right), \\
\mathbf{C}_{\Pi_{2}} & =(\mathbf{I}+\mathbf{K})\left(\boldsymbol{\Pi}_{1} \otimes \mathbf{U}_{1}\right)+\left(\mathbf{U}_{1} \otimes \boldsymbol{\Pi}_{1}\right) \\
& +(\eta-1)\left((\mathbf{I}+\mathbf{K})\left(\boldsymbol{\Pi}_{1} \otimes \mathbf{U}_{1}^{\prime}\right)+\left(\mathbf{U}_{1}^{\prime} \otimes \boldsymbol{\Pi}_{1}\right)\right) .
\end{aligned}
$$


Introducing these expressions into (68) and (69) gives after straightforward algebra manipulations

$$
\begin{aligned}
\mathrm{E}\left[\operatorname{Tr}\left(\delta \boldsymbol{\Pi}_{1, T} \mathbf{M}\right) \operatorname{Tr}\left(\delta \boldsymbol{\Pi}_{1, T} \mathbf{M}\right)\right] & \approx \frac{2}{T}\left(\left(\mathbf{a}^{H} \mathbf{U}_{1} \mathbf{a}\right)\left(\mathbf{a}^{H} \boldsymbol{\Pi}_{1} \mathbf{a}\right)+\operatorname{Re}\left[\left(\mathbf{a}^{H} \mathbf{U}_{2} \mathbf{a}^{*}\right)\left(\mathbf{a}^{T} \boldsymbol{\Pi}_{2}^{*} \mathbf{a}\right)\right]\right. \\
& \left.+(\eta-1)\left[\left(\mathbf{a}^{H} \mathbf{U}_{1}^{\prime} \mathbf{a}\right)\left(\mathbf{a}^{H} \boldsymbol{\Pi}_{1} \mathbf{a}\right)+\operatorname{Re}\left[\left(\mathbf{a}^{H} \mathbf{U}_{2}^{\prime} \mathbf{a}^{*}\right)\left(\mathbf{a}^{T} \boldsymbol{\Pi}_{2}^{*} \mathbf{a}\right)\right]\right]\right), \\
\mathrm{E}\left[\operatorname{Tr}\left(\delta \boldsymbol{\Pi}_{2, T}^{*} \mathbf{N}\right) \operatorname{Tr}\left(\delta \boldsymbol{\Pi}_{2, T} \mathbf{N}^{*}\right)\right] & \approx \frac{4}{T}\left(\left(\mathbf{a}^{H} \mathbf{U}_{1} \mathbf{a}\right)\left(\mathbf{a}^{H} \boldsymbol{\Pi}_{1} \mathbf{a}\right)+(\eta-1)\left(\mathbf{a}^{H} \mathbf{U}_{1}^{\prime} \mathbf{a}\right)\left(\mathbf{a}^{H} \boldsymbol{\Pi}_{1} \mathbf{a}\right)\right) .
\end{aligned}
$$

Incorporating expressions (66), (67), (70) and (71) into (65) proves (37). Then (38) follows with the same lines.

\section{Expressions of $\Pi, \mathbf{U}$ and $\mathbf{U}^{\prime}$}

The expression of $\boldsymbol{\Pi}, \mathbf{U}$ and $\mathbf{U}^{\prime}$ are derived from the two non-zero eigenvalues and the associated eigenvectors of the rank two matrix $\mathbf{S}=\sigma_{s}^{2}\left(\mathbf{a}_{1}, \mathbf{a}_{2}\right)\left(\begin{array}{c}\mathbf{a}_{1}^{H}+\rho \mathbf{a}_{2}^{H} \\ \rho^{*} \mathbf{a}_{1}^{H}+\mathbf{a}_{2}^{H}\end{array}\right)$. The non-zero eigenvalues of $\mathbf{S}$ are the roots of the quadratic polynomial: $\lambda^{2}-\operatorname{Tr}(\mathbf{S}) \lambda+\operatorname{det}\left[\sigma_{s}^{2}\left(\begin{array}{c}\mathbf{a}_{1}^{H}+\rho \mathbf{a}_{2}^{H} \\ \rho^{*} \mathbf{a}_{1}^{H}+\mathbf{a}_{2}^{H}\end{array}\right)\left(\mathbf{a}_{1}, \mathbf{a}_{2}\right)\right]$ which are $\lambda_{k}=\left\|\mathbf{a}_{k}\right\|^{2} \sigma_{s}^{2}\left(\alpha \pm \sqrt{\alpha^{2}-\gamma}\right), k=1,2$, with $\alpha \stackrel{\text { def }}{=} 1+\beta \operatorname{Re}(\rho)$ and $\gamma \stackrel{\text { def }}{=}\left(1-|\rho|^{2}\right)\left(1-\beta^{2}\right)$ where $\beta \stackrel{\text { def }}{=} \mathbf{a}_{1}^{H} \mathbf{a}_{2} / N^{2} \in[-1,+1]$ and the associated eigenvectors $\mathbf{v}_{k}=\left[N \sigma_{s}^{2}\left(1+\rho^{*} \beta\right)-\right.$ $\left.\lambda_{k}\right] \mathbf{a}_{1}-\left\|\mathbf{a}_{k}\right\|^{2} \sigma_{s}^{2}\left(\rho^{*}+\beta\right) \mathbf{a}_{2}$. Plugging these eigenvalues and eigenvectors in $\mathbf{U}=\left(\frac{\sigma_{n}^{2}}{\lambda_{1}}+\frac{\sigma_{n}^{4}}{\lambda_{1}^{2}}\right) \frac{\mathbf{v}_{1} \mathbf{v}_{1}^{H}}{\left\|\mathbf{v}_{1}\right\|^{2}}+\left(\frac{\sigma_{n}^{2}}{\lambda_{2}}+\frac{\sigma_{n}^{4}}{\lambda_{2}^{2}}\right) \frac{\mathbf{v}_{2} \mathbf{v}_{2}^{H}}{\left\|\mathbf{v}_{2}\right\|^{2}}$ and $\mathbf{U}^{\prime}=\frac{\sigma_{n}^{4}}{\lambda_{1}^{2}} \frac{\mathbf{v}_{1} \mathbf{v}_{1}^{H}}{\left\|\mathbf{v}_{1}\right\|^{2}}+\frac{\sigma_{n}^{4}}{\lambda_{2}^{2}} \frac{\mathbf{v}_{2} \mathbf{v}_{2}^{H}}{\left\|\mathbf{v}_{2}\right\|^{2}}$ and using $\boldsymbol{\Pi}=\mathbf{I}-\mathbf{A}\left(\mathbf{A}^{H} \mathbf{A}\right)^{-1} \mathbf{A}^{H}$, these three matrices are deduced after cumbersome but straightforward algebraic manipulations.

D. Expressions of $h_{1,1}, h_{2,1}, h_{1, m}, h_{2, m} h_{1, m}^{\prime}$ and $h_{2, m}^{\prime}$ for $U L A$

Substitution the general expressions of $\boldsymbol{\Pi}, \mathbf{U}$ and $\mathbf{U}^{\prime}$ given in Appendix $C$ into (35) yields, after some tedious algebraic manipulation and with the aid of symbolic algebra and calculus tools, that the terms involved in (39), (40) and (41) are 
given for arbitrary centrosymmetric arrays by,

$$
\begin{aligned}
h_{1,1} & =\frac{N-2}{1-|\rho|^{2}}, \\
h_{2,1} & =\frac{\eta(N-2)\left(1+|\rho|^{2}+2 \beta \operatorname{Re}(\rho)\right)}{N\left(1-\beta^{2}\right)\left(1-|\rho|^{2}\right)^{2}}, \\
h_{1, m} & =\frac{2}{|\rho|^{2}-1}\left(\frac{(N-2) \rho_{1, m}^{2}(\operatorname{Re}(\rho)-1)}{(1+\beta)^{2}}-\frac{g_{m}(1+\beta \operatorname{Re}(\rho))}{N\left(\beta^{2}-1\right)}\right) \\
h_{2, m} & =\frac{2 \eta}{\left(1-\beta^{2}\right)\left(|\rho|^{2}-1\right)^{2}}\left(\frac{(N-2) \rho_{1, m}^{2}\left(|\rho|^{2}(1+\beta)-2 \operatorname{Re}(\rho)^{2} \beta+(\beta-1)(2 \operatorname{Re}(\rho)-1)\right)}{N(\beta+1)^{2}}\right. \\
& \left.+\frac{g_{m}\left(|\rho|^{2}\left(1-\beta^{2}\right)+1+2 \operatorname{Re}(\rho)^{2} \beta^{2}+\beta^{2}+4 \beta \operatorname{Re}(\rho)\right)}{N^{2}\left(\beta^{2}-1\right)}\right), \\
h_{1, m}^{\prime} & =\frac{4(N-2)}{|\rho|^{2}-1}\left(\frac{\rho_{1, m} \rho_{1, m}^{\prime \prime}(\operatorname{Re}(\rho)-1)}{(\beta+1)^{2}}-\frac{\rho_{1, m}^{\prime 2}(\operatorname{Re}(\rho)+1)}{(\beta-1)^{2}}\right)-\frac{2(1+\beta \operatorname{Re}(\rho)) h_{m}}{N\left(\beta^{2}-1\right)\left(|\rho|^{2}-1\right)}, \\
h_{2, m}^{\prime} & =\frac{4(N-2)}{N\left(\beta^{2}-1\right)\left(|\rho|^{2}-1\right)^{2}}\left(\frac{\rho_{1, m}^{\prime 2}\left(|\rho|^{2}(\beta-1)-2 \operatorname{Re}(\rho)^{2} \beta-(\beta+1)(2 \operatorname{Re}(\rho)+1)\right)}{(\beta-1)^{2}}\right. \\
& \left.-\frac{\rho_{1, m} \rho_{1, m}^{\prime \prime}\left(|\rho|^{2}(1+\beta)-2 \operatorname{Re}(\rho)^{2} \beta+(\beta-1)(2 \operatorname{Re}(\rho)-1)\right)}{(\beta+1)^{2}}\right) \\
& -\frac{2 h_{m}\left(|\rho|^{2}\left(1-\beta^{2}\right)+1+\beta^{2}+2 \beta^{2} \operatorname{Re}(\rho)^{2}+4 \operatorname{Re}(\rho) \beta\right)}{N^{2}\left(\beta^{2}-1\right)^{2}\left(|\rho|^{2}-1\right)^{2}},
\end{aligned}
$$

with $g_{m} \stackrel{\text { def }}{=} g^{\operatorname{Alg} C_{C}}\left(\theta_{m}\right)=N\left(1-2 \frac{\rho_{1, m}^{2}}{1+\beta}\right)$ and $\left.h_{m} \stackrel{\text { def }}{=} \frac{d^{2} g^{\mathrm{Alg}_{\mathrm{C}}}(\theta)}{d \theta^{2}}\right|_{\theta=\theta_{m}}=4 N\left(\frac{\rho_{1, m}^{\prime 2}}{\beta-1}-\frac{\rho_{1, m}^{\prime \prime} \rho_{1, m}}{\beta+1}\right)$ and where $\beta=\frac{\mathbf{a}_{1}^{H} \mathbf{a}_{2}}{N}$, $\rho_{1, m}=\frac{\mathbf{a}_{1}^{H} \mathbf{a}_{m}}{N}, \rho_{1, m}^{\prime}=\frac{\mathbf{a}_{m}^{\prime} \mathbf{a}_{1}}{N}$ and $\rho_{1, m}^{\prime \prime}=\frac{\mathbf{a}_{m}^{\prime \prime} \mathbf{a}_{1}}{N}$.

\section{E. Expressions of $\mathbf{\Pi}_{1}, \mathbf{\Pi}_{2}, \mathbf{U}_{1}, \mathbf{U}_{2}, \mathbf{U}_{1}^{\prime}$ and $\mathbf{U}_{2}^{\prime}$}

The expression of $\widetilde{\boldsymbol{\Pi}}, \widetilde{\mathbf{U}}$ and $\widetilde{\mathbf{U}}^{\prime}$ are also derived from the two non-zero eigenvalues and the associated eigenvectors of the rank two matrix $\widetilde{\mathbf{S}}=\sigma_{s}^{2}\left(\widetilde{\mathbf{a}}_{1}, \widetilde{\mathbf{a}}_{2}\right)\left(\begin{array}{c}\widetilde{\mathbf{a}}_{1}^{H}+\rho^{\prime} \widetilde{\mathbf{a}}_{2}^{H} \\ \rho^{\prime} \widetilde{\mathbf{a}}_{1}^{H}+\widetilde{\mathbf{a}}_{2}^{H}\end{array}\right)$. The non-zero eigenvalues of $\widetilde{\mathbf{S}}$ are derived from the roots of the quadratic polynomial: $\lambda^{2}-\operatorname{Tr}(\widetilde{\mathbf{S}}) \lambda+\operatorname{det}\left[\sigma_{s}^{2}\left(\begin{array}{c}\widetilde{\mathbf{a}}_{1}^{H}+\rho^{\prime} \widetilde{\mathbf{a}}_{2}^{H} \\ \rho^{\prime} \widetilde{\mathbf{a}}_{1}^{H}+\widetilde{\mathbf{a}}_{2}^{H}\end{array}\right)\left(\widetilde{\mathbf{a}}_{1}, \widetilde{\mathbf{a}}_{2}\right)\right]$, which give the eigenvalues $\lambda_{1}=2 N \sigma_{s}^{2}(1-\beta)(1-\rho)$ and $\lambda_{2}=2 N \sigma_{s}^{2}(1+\beta)(1+\rho)$, and its associated eigenvectors are $\widetilde{\mathbf{v}}_{1}=\widetilde{\mathbf{a}}_{1}-\widetilde{\mathbf{a}}_{2}$ and $\widetilde{\mathbf{v}}_{2}=\widetilde{\mathbf{a}}_{1}+\widetilde{\mathbf{a}}_{2}$. This allows us to deduce the

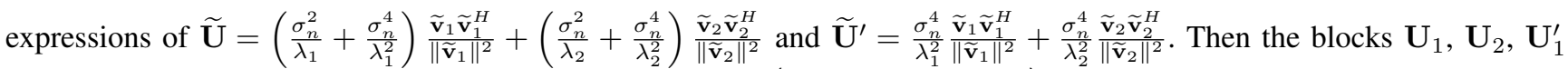
and $\mathbf{U}_{2}^{\prime}$ follow from the block decompositions of $\widetilde{\mathbf{v}}_{1} \widetilde{\mathbf{v}}_{1}^{H}=\left(\begin{array}{c}\mathbf{a}_{1} e^{i \phi_{1}}-\mathbf{a}_{2} e^{i \phi_{2}} \\ \mathbf{a}_{1}^{*} e^{-i \phi_{1}}-\mathbf{a}_{2}^{*} e^{-i \phi_{2}}\end{array}\right)\left(\mathbf{a}_{1}^{H} e^{-i \phi_{1}}-\mathbf{a}_{2}^{H} e^{-i \phi_{2}}, \mathbf{a}_{1}^{T} e^{i \phi_{1}}-\mathbf{a}_{2}^{T} e^{i \phi_{2}}\right)$ and $\widetilde{\mathbf{v}}_{2} \widetilde{\mathbf{v}}_{2}^{H}=\left(\begin{array}{c}\mathbf{a}_{1} e^{i \phi_{1}}+\mathbf{a}_{2} e^{i \phi_{2}} \\ \mathbf{a}_{1}^{*} e^{-i \phi_{1}}+\mathbf{a}_{2}^{*} e^{-i \phi_{2}}\end{array}\right)\left(\mathbf{a}_{1}^{H} e^{-i \phi_{1}}+\mathbf{a}_{2}^{H} e^{-i \phi_{2}}, \mathbf{a}_{1}^{T} e^{i \phi_{1}}+\mathbf{a}_{2}^{T} e^{i \phi_{2}}\right)$. Similarly the bocks $\boldsymbol{\Pi}_{1}$ and $\boldsymbol{\Pi}_{2}$ follow from $\widetilde{\mathbf{\Pi}}=\mathbf{I}-\widetilde{\mathbf{A}}\left(\widetilde{\mathbf{A}}^{H} \widetilde{\mathbf{A}}\right)^{-1} \widetilde{\mathbf{A}}^{H}$ with $\widetilde{\mathbf{A}}=\left(\begin{array}{cc}\mathbf{a}_{1} e^{i \phi_{1}} & \mathbf{a}_{2} e^{i \phi_{2}} \\ \mathbf{a}_{1}^{*} e^{-i \phi_{1}} & \mathbf{a}_{2}^{*} e^{-i \phi_{2}}\end{array}\right)$. 


\section{REFERENCES}

[1] H. Krim and M. Viberg, "Two decades of array signal processing research: the parametric approach," IEEE Signal Processing Mag., vol. 13, no. 4, pp. 67-94, 1996.

[2] Y. Zhou, D. Xu, W Tu, and C. Shi, ”Spatial information and angular resolution of sensor array," Signal Processing, vol. 174, Sept. 2020 .

[3] W. Wang, S.Yan, and L. Mao, On the statistical performance of spherical harmonics MUSIC,” Signal Processing, vol. 174, Sept. 2020.

[4] H. Cox, "Resolving power and sensitivity to mismatch of optimum array processors," J. Acoust. Soc. America, vol. 54, no. 3, pp. 771-785, 1973.

[5] K.C. Sharman and S.T. Durrani, "Resolving power of signal subspace methods for finite data lengths," in Proc. ICASSP, Tampa, Florida, April 1985.

[6] M. Kaveh and A.J. Barabell, "The statistical performance of the MUSIC and the Minimum-Norm algorithms in resolving plane waves in noise," IEEE Trans. on ASSP, vol. 34, no. 2, pp. 331-341, April 1986.

[7] M. Kaveh and H. Wang, "Threshold properties of narrowband signal subspace array processing methods," in Advances in Spectrum Analysis and Array Processing, S. Haykin, Prentice-Hall, pp. 173-220, vol. 2, 1991.

[8] C. Zhou, F. Haber, and D.L. Jaggard, "A resolution measure for the MUSIC algorithm and its application to plane wave arrivals contamined by coherent interference," IEEE Trans. on Signal Processing, vol. 39, no. 2, pp. 454-463, February 1991.

[9] S.U. Pillai and G.H. Kwon, "Performance analysis of MUSIC-type high resolution estimators for direction finding in correlated and coherent scenes," IEEE Trans. on ASSP, vol. 37, no. 8, pp. 1176-1189, August 1989.

[10] H.B. Lee and M.S. Wengrovitz, "Resolution threshold of beamspace MUSIC for two closely spaced emitters," IEEE Trans. Signal Process., vol. 38, no. 9, pp. 1445-1559, Sept. 1990.

[11] P. Foster, E. Villier, "Simplified formulas for performance analysis of MUSIC and Min Norm," in Proc. OCEANS'98, Nice, Sept. 1998.

[12] P. Foster, E. Villier, Performances asymptotiques des méthodes haute résolution in S. Marcos Editor, Les méthodes à haute résolution, Hermes, 1998.

[13] H. Abeida and J.-P. Delmas, ”Statistical performance of MUSIC-like algorithms in resolving noncircular sources," IEEE Trans. Signal Process., vol. 56, no. 9, pp. 4317-4329, Sept. 2008.

[14] M. Shahram and P. Milanfar, "On the resolvability of sinusoids with nearby frequencies in the presence of noise," IEEE Trans. Signal Process. vol. 53, no. 7, pp 2579-2588, July 2005.

[15] Z. Liu and A. Nehorai, "Statistical angular resolution limit for point sources," IEEE Trans. Signal Process. vol. 55, no. 11, pp. 5521-5527, Nov. 2007.

[16] A. Amar and A. J. Weiss, "Fundamental limitations on the resolution of deterministic signals," IEEE Trans. Signal Process. vol. 56, no. 11, pp. 5309-5318, August 2008.

[17] H. B. Lee, 'The Cramer-Rao bound on frequency estimates of signals closely spaced in frequency," IEEE Trans. Signal Process., vol. 40, no. 6, pp. 1507-1517, June 1992.

[18] S.T. Smith, "Statistical resolution limits and the complexified Cramer-Rao bound," IEEE Trans. Signal Process., vol. 53, no. 5, pp. 1597-1609, May 2005.

[19] J.-P. Delmas and H. Abeida, "Statistical resolution limits of DOA for discrete sources," in Proceedings of ICASSP, Toulouse, France, April 2006.

[20] M. N. El Korso, R. Boyer, A. Renaux, and S. Marcos, "Statistical resolution limit of the uniform linear cocentered orthogonal loop and dipole array," IEEE Trans. Signal Process., vol. 59, no. 1, pp. 425-431, Jan. 2011. 
[21] X. Zhang, M. N. El Korso, and M. Pesavento, "Angular resolution limit for deterministic correlated sources," Proc. of ICASSP, Vancouver, Canada, May 2013.

[22] X. Zhang, M.N.El Korso, and M. Pesavento, "MIMO radar performance analysis under K-distributed clutter," in Proc. of ICASSP, Florence, Italy 2014.

[23] D.T. Vu, M. N. El Korso, R. Boyer, A. Renaux, and S. Marcos, ”Angular resolution limit for vector-sensor arrays: detection and information theory approaches," in IEEE SSP Workshop, Nice, France, June 2011.

[24] E. Conte, M. Longo, and M. Lops, "Modelling and simulation of non-Rayleigh radar clutter," in Proc. Inst. Electr. Eng. F, vol. 138, no. 2, pp. 121-130, 1991.

[25] M. Sekine, S. Ohatani, T.Musha, T. Irabu, E. Kiuchi, T. Hagisawa, and Y. Tomita, ”Weibull-distributed ground clutter," IEEE Trans. Aerosp. Electron. Syst., vol. 17, no. 4, pp. 596-598, 1981.

[26] D. Middleton, "Man-made noise in urban environments and transportation systems: Models and measurements," IEEE Trans. Commun., vol. 21, no. 11, pp. 1232-1241, 1973.

[27] D. B. Williams and D. H. Johnson, "Robust estimation of structured covariance matrices," IEEE Trans. Signal Process., vol. 41, no. 9, pp. 2891-2905, 1993.

[28] E. Ollila, D. Tyler, V. Koivunen, and H. Poor, "Complex elliptically symmetric distributions: Survey, new results and applications," IEEE Trans. Signal Process., vol. 60, no. 11, pp. 5597-5625, Nov. 2012.

[29] H. Abeida and J.-P. Delmas, "Efficiency of subspace-based estimators for elliptical symmetric distributions," Signal Processing, vol. 174, Oct. 2020 .

[30] H. Abeida and J.-P. Delmas, "Slepian-Bangs formula and Cramer Rao bound for circular and non-circular complex elliptical symmetric distributions," IEEE Signal Process. Letters, vol. 26, no. 10, pp. 1561-1565, Oct. 2019.

[31] H. Abeida and J.-P. Delmas, "Robustness of subspace-based algorithms with respect to the distribution of the noise: Application to DOA estimation," Signal Processing, vol. 164, pp. 313-319, June 2019.

[32] S. Visuri, H. Oja, and V. Koivunen, "Subspace-based direction of arrival estimation using nonparametric statistics," IEEE Trans. Signal Process., vol. 49, no. 9, pp. 2060-2073, Sept. 2001.

[33] S. Fortunati, F. Gini, and M. S. Greco, "Semiparametric stochastic CRB for DOA estimation in elliptical data model," Proceedings of EUSIPCO, A Coruna, Spain, Sept. 2019.

[34] R. Maronna, "Robust $M$-estimators of multivariate location and scatter," Annals of Statistics, vol. 4, no. 1, pp. 51-67, Jan. 1976.

[35] E. Ollila and V. Koivunen, "Robust antenna array processing using $M$-estimators of pseudo covariance," 14th lntermational Symposium on Personal lndoor and Mobile Radio Communication, 2003.

[36] D. E. Tyler, "A distribution-free estimator of multivariate scatter," The Annals of Statistics vol. 615 no. 21 pp. $234-251,1987$.

[37] F. Pascal, P. Forster, J.-P. Ovarlez, and P. Larzabal, "Performance analysis of covariance matrix estimates in impulsive noise," IEEE Trans. Signal Process., vol. 56, no. 6, 2018, pp. 2206-2217, June 2008.

[38] H. Krim, P. Forster, and G. Proakis, "Operator approach to performance analysis of root-MUSIC and root-min-norm," IEEE Trans. Signal Process., vol. 40, no. 7, pp. 1687-1696, July 1992.

[39] P. Gounon, C. Adnet, and J. Galy, "Localization angulaire de signaux non circulaires," Traitement du Signal, vol. 15, no. 1, pp. 17-23, 1998.

[40] H. Abeida and J.-P. Delmas, "Robustness and performance analysis of subspace-based DOA estimation for rectilinear correlated sources in CES data model," Signal Processing, vol. 178, Jan. 2021.

[41] D. E. Tyler, "Radial estimates and the test for sphericity," Biometrika, vol. 69, no. 2, pp. 429-436, 1982.

[42] M. Mahot, F. Pascal, and F. Foster, and J.-P. Ovarlez, "Asymptotic properties of robust complex covariance matrix estimates," IEEE Trans. Signal Process., vol. 61, no. 13, 2018, pp. 3348-3356, July 2013. 
[43] H.B. Lee and M.S. Wengrovitz, "Statistical characterization of the MUSIC algorithm null spectrum," IEEE Trans. on ASSP, vol. 39, no. 6, pp. 1333-1347, June 1991.

[44] F. Roemer and M. Haardt, "Using 3D unitary ESPRIT on a hexagonal shaped ESPAR antenna for 1D and 2D DOA estimation," in IEEE ITG workshop on smart antennas, Duisburg, Germany, April 2005.

[45] P. Stoica and A. Nehorai, "MUSIC, maximum likelihood, and Cramer-Rao bound: further results and comparisons," IEEE Trans. ASSP, vol. 3, no. 12, pp. 2140-2150, Dec. 1990.

[46] A. J. Weiss and B. Friedlander, "On the Cramer-Rao bound for direction finding of correlated signals," IEEE Trans. Signal Process., vol. 41, no. 1, pp. 495-499, Jan. 1993.

[47] H. Abeida and J.-P. Delmas, "MUSIC-like estimation of direction of arrival for non-circular sources," IEEE Trans. Signal Process., vol. 54, no. 7, pp. 2678-2690, July 2006.

[48] D.A. Harville, Matrix algebra from a statistical's perspective, Springer-Verlag, 1999. 